\title{
BEOBACHTUNGEN UND ÜBERLEGUNGEN ZUR EISZEITLICHEN DEPRESSION VON SCHNEEGRENZE UND STRUKTURBODENGRENZE IN DEN TROPEN UND SUBTROPEN
}

\author{
Mit 8 Abbildungen z. T. als Beilage VII, 6 Fotos und 1 Schema
}

\section{Klaus Heine}

Summary: Observations and considerations concerning depression of the snow line and boundary of polygonal soils in the tropics and sub-tropics.

The discussion concerning the convergence or divergence of the snow line and boundary of polygonal soils in the sub-tropics is the result of observing forms rather than processes. The same forms may arise from different processes.

In the case of the last ice age several depressions of the snow line can be established in the sub-tropics and tropics, although these, however, did not run concurrently.

It is necessary to start out from the assumption that the relationship of the snow line position, the permafrost boundary and the polygonal soil boundary was not constant during the late Quaternary, since fluctuations in the hygric climate occurred independently of thermal fluctuations. This holds good for the sub-tropics on the one hand and for the tropics on the other.

In this connection attention should be drawn to the problems arising in large areas from reconstructions - frequently attempted - of snow lines and polygonal soil boundaries of the last glacial period, since phenomena of unequal age are employed in these reconstructions.

In jüngster Zeit wird immer häufiger versucht, die eiszeitlichen Zirkulationsverhältnisse weltweit zu rekonstruieren (BARRY \& Williams 1975). Für derartige Betrachtungen und Simulationen werden verschiedene Quellen ausgewertet; dabei spielen die horizontale und vertikale Ausdehnung der pleistozänen Eismassen, die eiszeitlichen Temperaturen der Meeresoberfläche, eiszeitliche Albedoveränderungen infolge veränderter Vegetationsverteilung, eiszeitliche Verschiebungen der Küstenlinien als Folge der Meeresregression und Hinweis auf Permafrost sowie auf periglaziale Bedingungen eine besondere Rolle. Der Verlauf der Strukturbodengrenze und der Schneegrenze während des Maximums der letzten Eiszeit ist gerade für die tropischen und subtropischen Gebiete, aus denen bisher oft noch wenig Indizien für pleistozäne Klima- und Vegetationsänderungen vorliegen, herangezogen worden, um globale eiszeitliche Zirkulationsmuster zu rekonstruieren. Bisher war es jedoch nur selten möglich, die eiszeitliche Depression der Strukturbodengrenze wie auch der Schneegrenze zeitlich genau zu erfassen; außertropische Gletschervorstöße wurden mit subtropischen Moränen und „Pluvial“-Seen und diese wieder mit tropischen Gletschervorstößen korreliert. Stillschweigend wurde eine Gleichaltrigkeit der Vorgänge und Formen angenommen. Sollte sich jedoch herausstellen, daß dieser vermutete Synchronismus der Ereignisse nicht immer zutrifft, so hat das weitreichende Konsequenzen im besonderen für die Paläoklimatologie, Paläogeographie und Paläoökologie.

Einige Beobachtungen und Überlegungen zur Strukturbodengrenze und deren eiszeitliche Absenkung und zur pleistozänen Schneegrenzdepression, die einerseits auf differenziertere Vorstellungen und andererseits auf Lösungen der Probleme hinweisen, sollen im folgenden mitgeteilt werden.

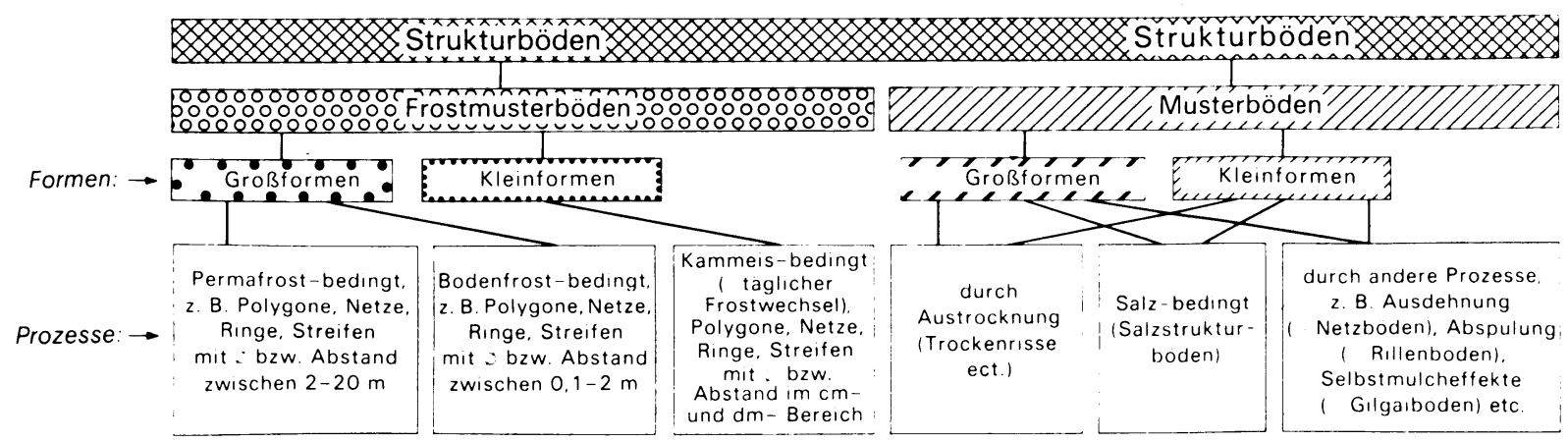

Schema: Genetische Unterscheidung verschiedener „Strukturböden“

Genetic differentiation of various polygonal soils 


\section{Strukturbodengrenzen}

Der Begriff Strukturbodengrenze wird im folgenden wie bei HöllermanN (1972 b) benutzt, nämlich im Zusammenhang mit Strukturböden (Frostmusterböden), die augenfällig an kaltklimatische Bedingungen frostdynamischer Art gebunden sind; der Begriff "Strukturbodengrenze“ mit Gänsefüßchen umfaßt alle "Strukturböden“, wie im folgenden Schema angegeben.

Die sogenannte „periglaziale Höhenstufe" tropischer, subtropischer wie auch außertropischer Gebirge wird oft in ein oberes und unteres Stockwerk unterteilt (HöllermanN 1976 a); das obere Stockwerk befindet sich $\mathrm{zwischen} \mathrm{der} \mathrm{klimatischen} \mathrm{Schneegrenze}$ und der Strukturbodengrenze; zwischen der Strukturbodengrenze und der unteren "Solifluktionsgrenze“ liegt das untere Stockwerk der „periglazialen Höhenstufe“. Die Strukturbodengrenze verbindet in einem größeren Untersuchungsgebiet - oder weltweit - die untersten Vorkommen von Frostmusterböden.

Eine umfangreiche Literatur über „Strukturbodenvorkommen" aus allen Kontinenten zeigt, daß diese Phänomene - die zumeist einem periglazialen Formenschatz zugeordnet werden - auch weit abseits des rezenten Periglazialbereichs auftreten können (HöLLERMANN 1976 b), wie zum Beispiel gestreifte Auffrierböden bei Bonn in $125 \mathrm{~m}$ NN oder an randtropischen zentralmexikanischen Vulkanen in $2750 \mathrm{~m}$ NN (die obere Waldgrenze liegt dort in ca. $4000 \mathrm{~m}$ $\mathrm{NN}$ ) belegen. Diese „unter besonderen Bedingungen in Abhängigkeit vom Witterungsgeschehen kurzfristig entstandenen Frostbodenformen " (HöllermanN 1976 b) haben dazu geführt, daß die Strukturbodengrenze unterschiedlich definiert wird. Werden alle bisher bekannten "Strukturböden“ (Schema) berücksichtigt, so ist die Strukturbodengrenze in vielen Gebieten (wie z. B. in Europa) ohne Aussagekraft; in den Subtropen (z. B. Sahara) scheinen Frostbodenformen weit unterhalb der Schneegrenze unter besonderen Bedingungen gebildet $\mathrm{zu}$ werden, weshalb von einem Divergieren der Schnee- und Strukturbodengrenze in den Subtropen gesprochen wird (HöverMANN 1954, 1962). Werden nur die untersten größeren Vorkommen von rezenten Frostmusterböden wobei von vereinzelten tieferliegenden abgesehen wird - bei der Festlegung der Strukturbodengrenze hinzugezogen, so sieht ihr Verlauf in Bezug zur Schneegrenze wieder anders aus (vgl. Graf 1973).

Aus der Diskussion über den Verlauf der Strukturbodengrenze geht deutlich hervor, daß die Betrachtung der Formen hier nicht weiterführt. Nur die Berücksichtigung der die "Strukturböden" verursachenden Prozesse ermöglicht weiterführende Aussagen, denn gleiche Formen können durch verschiedene Vorgänge gebildet werden. In den Subtropen und Tropen kann infolge Kammeisbildung ein scheinbar "periglazialer Formenschatz" häufig weit unter- halb der Schneegrenze entstehen. Werden kammeisbedingte Formen als rezente periglaziale Kleinformen angesehen, dann muß heute von einem Divergieren der Schnee- und Strukturbodengrenze in den Subtropen gesprochen werden; werden die kammeisbedingten subtropisch-tropischen Strukturbodenformen nicht den außertropischen Frostbodenformen gleichgesetzt, die durch Bodenfrost (d. h. Temperaturen unter dem Gefrierpunkt dringen mehrere Dezimeter in den Boden ein) entstanden sind, so zeigt die Höhenlage der frostbodenbedingten Formen in den Subtropen ein Konvergieren der Schnee- und Strukturbodengrenze (HEINE 1977).

Der Begriff der Solifluktion (vgl. BENEDICT 1976) ist in der deutschsprachigen Literatur nicht eindeutig auf bestimmte Prozesse festgelegt. Strukturbodenformen sowohl der gebundenen als auch der halbgebundenen oder freien Solifluktion können einerseits durch - zumeist jahreszeitlichen - Bodenfrost mit oder ohne Permafrost, andererseits durch wiederholtes tägliches Kammeisauffrieren entstehen. Grundverschiedene Prozesse, nämlich Kammeisauffrieren, Frostkriechen und Gelisolifluktion, erzeugen Strukturböden (vgl. WASHBURN 1973). Sieht man die Literatur hinsichtlich der in Frage kommenden Prozesse durch, so stellt sich heraus, daß die meisten „periglazialen“ Formen in den Subtropen und Tropen - soweit das anhand der Berichte und Bilder festzustellen möglich ist - kammeisbedingte Formen darstellen; sie können bei Vorhandensein entsprechender Bodenfeuchte überall dort auftreten, wo gelegentlich noch Temperaturen unter dem Geírierpunkt erreicht werden. Günstig für ihre Bildung sind allophanreiche vulkanische Böden, da diese die Kammeisbildung infolge bestimmter Werte der Bodenwasserspannung erleichtern. Beispiele für kammeisbedingte "periglaziale“ Formen in den Tropen und Subtropen finden sich u. a. bei Hastenrath (1973 a) und Furrer \& Freund (1973) für den Mt. Kenya und Kilimandjaro, bei Graf (1973) für Bolivien und Peru, bei HöllermanN (1972 a, 1976 a) für die Sierra Nevada/USA und Teneriffa, bei HövermanN (1954) für Erithrea und Nordäthiopien, bei Hagedorn (1974) für die Sinai-Gebirge, bei LÖffler (1975) für Papua Neuguinea, bei Schubert (1975) für Venezuela, bei Heine (1977) für Mexiko und bei Troll (1944) für Südamerika und Afrika.

Troll hat 1944 als erster auf den planetarischen Wandel des Periglazialklimas und dessen Ausprägung in der unterschiedlichen Ausbildung der Frostmusterböden hingewiesen (vgl. HöllermanN 1976 a); seither werden subtropisch-tropische kammeisbedingte Miniaturstrukturbodenformen mit außertropischen bodenfrostbedingten Formen verglichen; hier sollte aber - um Mißverständnisse auszuschließen - eine genaue Unterscheidung getroffen werden.

In Abb. 1 sind verschiedene Prozesse, die „Strukturböden “ im randtropischen Mexiko bewirken können, schematisch in ihrer Verbreitung dargestellt. Ver- 


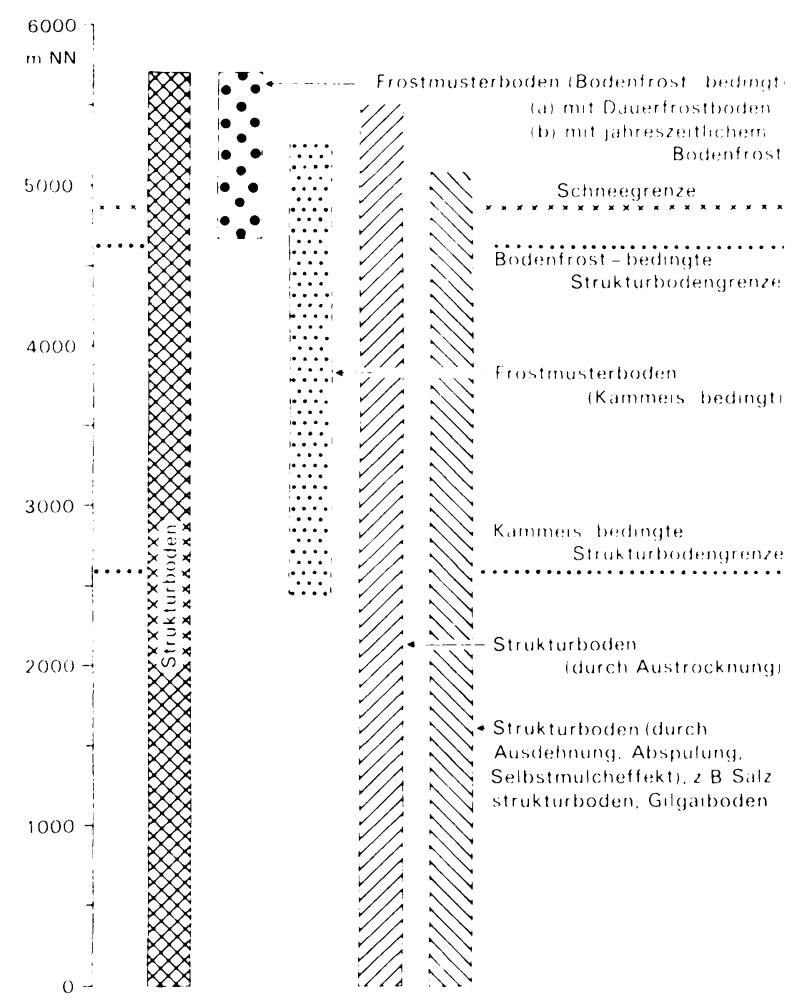

$A b b$. 1: Schematische Darstellung der vertikalen Verbreitung genetisch verschiedener Strukturböden im randtropischen Mexiko. Da gleiche Formen durch verschiedene Prozesse gebildet werden können, läßt sich eine einzige „untere Strukturbodengrenze" nicht festlegen.

Schematic presentation of the vertical distribution of genetically different polygonal soils on the fringe of the tropics in Mexico. As different processes may produce the same forms, a single "lower limit of polygonal soils" cannot be established.

schiedene Vorgänge sind dafür verantwortlich, daß "Strukturböden“ in allen Höhenbereichen zwischen dem Meeresspiegel und den höchsten eisfreien Bergflanken (bis ca. $5700 \mathrm{~m}$ ) vorkommen können. Dauerfrostboden (HEINE 1975 a) und jahreszeitlicher Bodenfrost, der einige Zentimeter bis Dezimeter in den Boden eindringen kann, können Strukturböden erzeugen, die als Frostmusterböden in die Literatur eingegangen sind (BREMER 1965; WASHBURN 1973). Steinringe, -polygone, -netze und -streifen mit Materialsortierung, die einen Durchmesser bzw. Abstand von $2-20 \mathrm{~m}$ aufweisen, werden nur in Gebieten beobachtet, in denen ein nahezu zusammenhängender Dauerfrostboden existiert. Kleinere Formen (unter $1 \mathrm{~m}$ ) werden häufig dort gebildet, wo alljährlich der Frost $0,1-2 \mathrm{~m}$ in den Boden eindringt (vgl. GoldTHWAIT 1976). Miniaturformen im Zentimeter-, höchstens im Dezimeterbereich sind an tageszeitliche Frostwechsel geknüpft und in der Regel mit Kammeisbildungen verbunden. Aus den Tropen und Subtropen werden fast ausschließlich Miniaturformen beschrieben. Permafrost- und bodenfrostbedingte Formen sind mir aus den Gebirgen der Feuchttropen nicht bekannt geworden, lediglich im rand- und subtropischen Bereich lassen sie sich mitunter finden, so auch in Mexiko (Foto 1 und 2). Ihre Verbreitung in den Tropen und Subtropen ist an die Gebiete gebunden, die zwischen der Schneegrenze und der unteren Permafrostgrenze bz.w. der Untergrenze \pm regelmäßig auftretender jahreszeitlicher Bodenfröste liegen (vgl. Graf 1976). Gebiete mit diesen klimatischen Eigenschaften treffen wir häufig in den Randtropen und Subtropen mit winterlicher Aridität an; in den Feuchttropen dagegen liegt die Permafrostgrenze über der Schneegrenze (HEINE 1975 a); in den feuchten Rand- und Subtropen verhindert oft eine winterliche Schneedecke das Eindringen des Frostes in den Boden.

In den extrem (winter-)ariden Gebieten fehlen die Frostmusterböden, denn bekanntlich bedarf es zu ihrer Bildung einer gewissen Durchfeuchtung des Materials. In Mexiko sind überall dort die Bedingungen für die Entstehung von Frostmusterböden i.e. S. günstig, wo auch im zumeist trockenen Winterhalbjahr genügend Feuchtigkeit vorhanden ist, nämlich beispielsweise unmittelbar unterhalb rezenter Gletscherzungen. Die in Mexiko beobachteten Frostmusterböden (Foto 1 und 2) sind an fossile Dauerfrostboden-Inseln gebunden, die im Bereich der nach ca. 1890 gletscherfrei gewordenen Hänge auftreten (HeINE 1975 a). Damit ist auch gleichzeitig das maximale Alter dieser Frostmusterböden auf rund 80 Jahre festgelegt; viele Frostmuster befinden sich in Höhen, die erst vor wenigen Jahrzehnten vom abschmelzenden Gletscher freigegeben wurden $\left.{ }^{1}\right)$.

Miniaturfrostmusterböden (Foto 2 und 3), die kammeisbedingt sind (HEINE 1977), können an den Hängen mexikanischer Vulkangebirge sehr häufig bei feuchten Bodenbedingungen und tageszeitlichen Frostwechseln in Höhen über $3000 \mathrm{~m}$ beobachtet werden; unter günstigen klimatischen Kammeisbildungsbedingungen findet man Miniatur-Auffriermusterböden auch in tieferen Lagen. Ihre Bildung ist weder an Permafrost noch jahreszeitlichen Frostwechsel gebunden, worauf bereits TROLL (1944) nachdrücklich hingewiesen hat. Allerdings kommen gleiche Formen auch in absolut frostfreien Gebieten unter Sonderbedingungen vor, wie die Beobachtungen von SCHUBERT (1974 c) auf der Insel La Orchila (Kleine Antillen) bezeugen.

Neben den bodenfrost- und kammeisbedingten Strukturböden werden Musterböden in Mexiko wie auch anderswo durch Austrocknung (Foto 4) und verschiedene andere Prozesse hervorgerufen, auf die hier im einzelnen nicht eingegangen werden soll; Gilgai-

1) Steinstreifen können auch das Ergebnis von Schmelzwasser-Ausspülungsvorgängen sein, nicht jedoch die horizontal liegenden Steinnetze. 

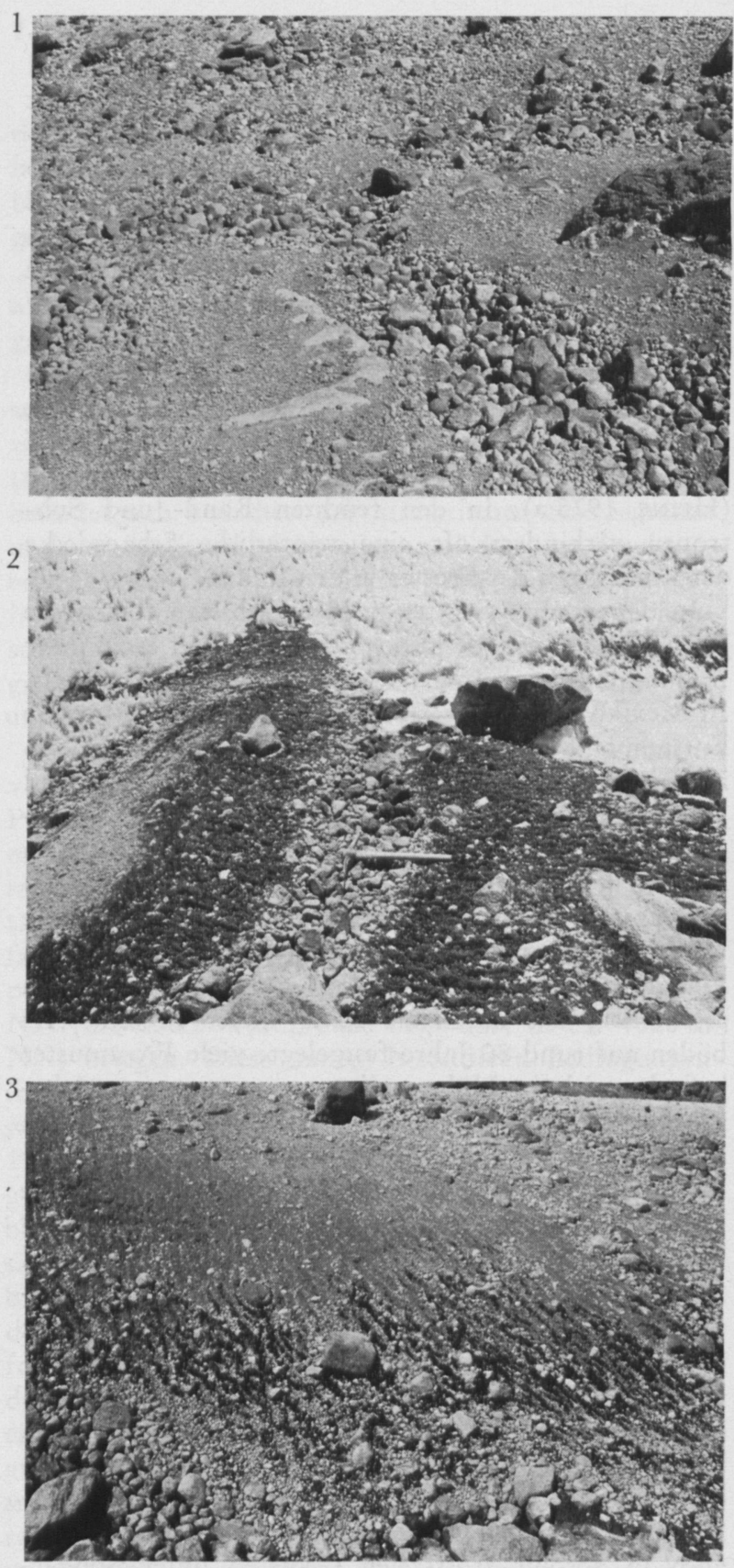

Foto 1: Steinringe am Nordhang des Pico de Orizaba in rund $4850 \mathrm{~m}$ Höhe. Der Durchmesser der Formen beträgt etwa $1,5 \mathrm{~m}$. Die Steinringe sind über einem fossilen Dauerfrostboden ausgebildet. Aufnahme: HeIne, 1. III. 1975.

Stone rings on the northern slope of the Pico de Orizaba at an altitude of about $4850 \mathrm{~m}$. The diameter of the forms measures about $1.5 \mathrm{~m}$. The stone rings are formed over fossil permafrost soil.

Foto 2: Steinstreifen am Pico de Orizaba in rund $4850 \mathrm{~m}$ Höhe (Nordhang). Die Steinstreifen - im linken und rechten Bildteil werden die Streifen vom Büßerschnee be-
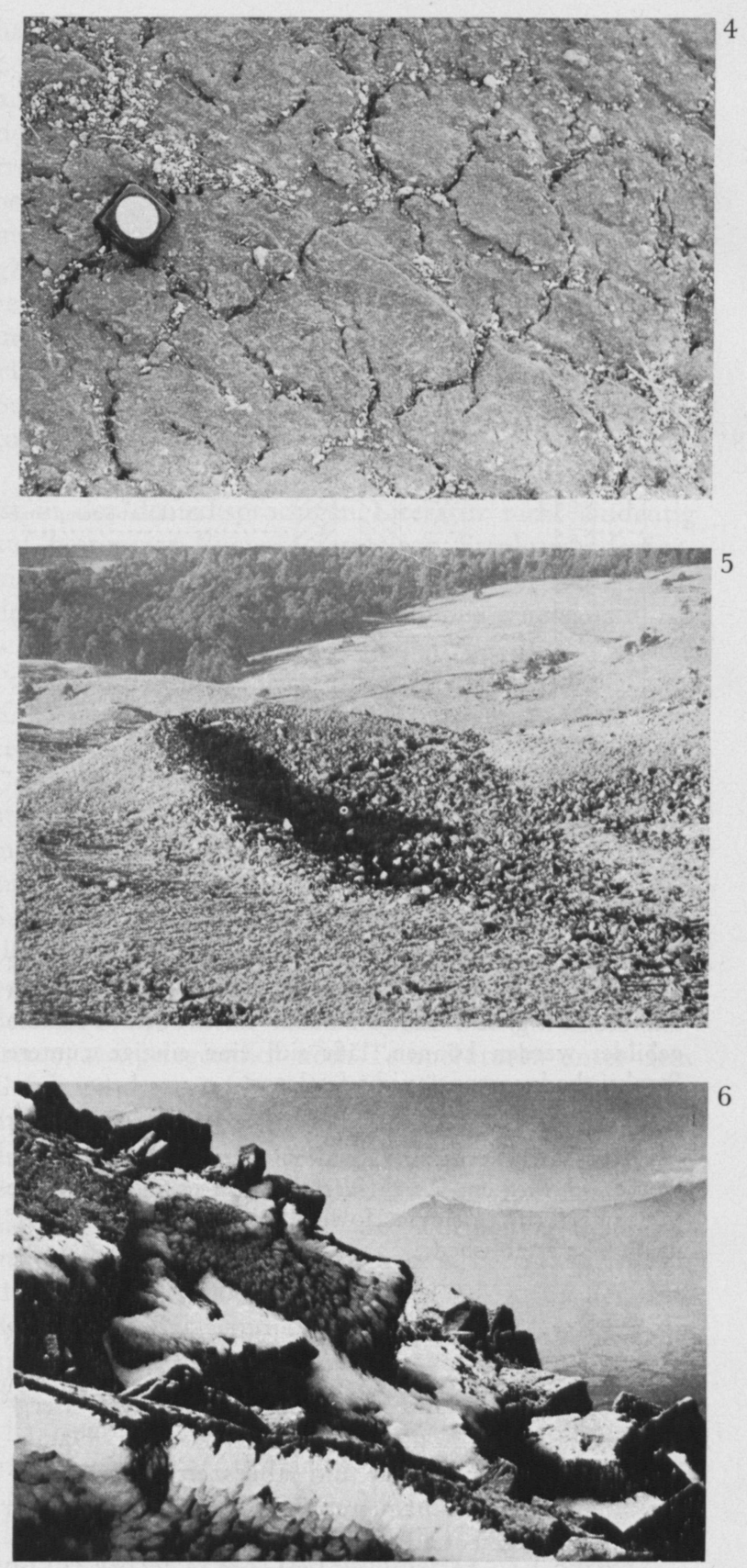

deckt - sind über einem fossilen Dauerfrostboden ausgebildet. Im feuchten Feinmaterial zwischen den Steinstreifen sind kammeisbedingte Ost-West-gerichtete (= senkrecht zu den hangabwärts verlaufenden Steinstreifen) Miniaturstreifenböden zu sehen. Aufnahme: HeINE, 1. III. 1975.

Stone stripes on the Pico de Orizaba at an altitude of about $4850 \mathrm{~m}$. (north slope). The stone stripes are formed over a fossil permafrost soil - in the righthand and lefthand parts of the picture the stripes are covered by Büsserschnee. In the damp, fine material between the stone stripes miniature stripe soils are to be seen, which are due to needle ice and lie in an east-west direction 
böden (BeCKMANN et al. 1973; BREMER 1965) und Salzstrukturböden (HölleRMANN 1972 b; WASHBURN 1973) können in den Tropen und Subtropen auch in d e n Höhenlagen gebildet werden, wo man Strukturböden für die Festlegung der periglazialen Strukturbodengrenze sucht (vgl. HastenRATH 1976).

Ein Schema des planetarischen Wandels der die Frostmusterböden bewirkenden Prozesse ist in Abb. 2 dargestellt. Aus ihr geht hervor, daß die Gebiete mit einem sehr häufigen Auftreten von kammeisbedingten Miniaturstrukturböden in den Feuchttropen zu suchen sind; dieser Umstand resultiert daraus, daß die Anzahl der Frostwechseltage bei gleichzeitig feuchten Bodenverhältnissen mit günstigen kammeisbedingenden bodenphysikalischen Eigenschaften (z. B. vulkanische Böden) unterhalb der Schneegrenze in den Feuchttropen am größten ist. Nähert man sich den wintertrockenen Randtropen, so sind durch die zeitlich bedingten trockenen Bodenverhältnisse die Voraussetzungen für Kammeisbildungen nur noch zeitweise gegeben, weshalb Miniaturstrukturböden weniger häufig beobachtet werden können (MESSERLi 1973). In extrem ariden Gebieten können - selbst bei häufigen Frostwechseltagen - Miniaturstrukturböden nur nach gelegentlichen Niederschlägen auftreten. Allerdings zeigen die Befunde aus extrem ariden Gebieten, daß Frostwechsel auch in relativ niedrigen Höhen vorkommt (JANNSEN 1972), was bei günstigen Bodenwasserverhältnissen in entsprechenden Substraten zu kurzlebigen Miniaturstrukturböden führen kann.
In den polwärtigen Gebieten der Subtropen, die in entsprechenden Höhenlagen regelmäßig jahreszeitliche Frostwechsel mit z. T. tiefreichendem Bodenfrost aufweisen, treten neben die Miniaturstrukturböden auch bodenfrostbedingte Frostmusterböden mit größeren Mustern (vgl. Höllermann 1972 b). Diese Phänomene sind es, die aus den gemäßigten und polaren Breiten in der Regel als Frostmusterböden beschrieben werden (Trolls außertropische Großformen). Vereinzelt kommen diese Großformen aber auch in den Subtropen und Randtropen vor, so daß eine Überlappung beider auf unterschiedliche Bildungsbedingungen zurückgehende Frostmusterböden (Foto 2 und 3) vorkommen kann (Heine 1977; GrAF 1976).

Während bodenfrost- und kammeisbedingte $\mathrm{re}$ $\mathrm{z}$ e n te Formen gleichermaßen gut beobachtet werden können (kammeisbedingte Formen bilden sich und vergehen oft in Stunden oder Tagen; sie kommen aber häufig vor), werden fossile Strukturbodenformen in der Regel nur aus der Gruppe der bodenfrostbedingten Formen noch erkannt, da die kammeisbedingten (Miniatur-)Formen zumeist keine lange Lebensdauer haben und auch als fossile Formen $-z$. B. unter einer jüngeren Sedimentdecke im Profil - meines Wissens bisher nirgends in den Tropen und Subtropen beobachtet wurden.

Daraus folgt, daß die Rekonstruktionen der eiszeitlichen Depression der Strukturbodengrenze stets die bodenfrostbedingten Formen berücksichtigen und nicht die kammeisbedingten. Werden daher in den Sub-
(= vertical to the downslope direction of the stone stripes).

Foto 3: Hangabwärts gerichtete, sortierte Miniaturstreifen. Die Formen sind kammeisbedingt und befinden sich in rund $4850 \mathrm{~m}$ Höhe am Pico de Orizaba. Der Abstand der Streifen beträgt maximal $0,15 \mathrm{~m}$. Aufnahme: Heine, 1. III. 1975.

Sorted miniature stripes, directed downslope. The forms are due to needle ice and are found on the Pico de Orizaba at an altitude of about $4850 \mathrm{~m}$. The interval between stripes amounts to a maximum of $0.15 \mathrm{~m}$.

Foto 4: Miniaturstrukturboden am Paso de Cortés (Sierra Nevada, Mexiko) in rund $4000 \mathrm{~m}$ Höhe. In den Trockenrissen des Andosols werden die vom Wind transportierten Bimslapilli z. T. festgehalten, so daß eine Materialsortierung vorgetäuscht wird. Die Streifung von links oben im Bild nach rechts unten ist windbedingt. Kantenlänge des Höhenmessers: $6 \mathrm{~cm}$. Aufnahme: Heine, 20. II. 1975.

Miniature polygonal soil on the Paso de Cortés (Sierra Nevada, Mexico) at about $4000 \mathrm{~m}$. altitude. Some of the pumice lapilli transported there by the wind are retained in the mud cracks of the andosol, so that sorting of materials is feigned. The striping which runs from the top lefthand to the bottom righthand corner of the illu- stration is due to wind. The edge of the altimeter measures $6 \mathrm{~cm}$.

Foto 5: Nivationsnische am Südhang des Nevado de Toluca in rund $4200 \mathrm{~m}$ Höhe. Die größte relative Höhe des sichelförmigen Walles beträgt $15 \mathrm{~m}$. Die Nivationsnische wurde vermutlich in der „Kleinen Eiszeit" gebildet. Aufnahme: HeINE, 16. III. 1975.

Nivation niche on the southern slope of the Nevado de Toluca at an altitude of about $4200 \mathrm{~m}$. The greatest relative height of the sickle-shaped mound is $15 \mathrm{~m}$. The nivation niche was presumably formed in the "Little Ice Age".

Foto 6: Eisbesatz am kantigen Blockschutt des MalincheGipfels in ca. $4450 \mathrm{~m}$ Höhe. Die Eis-Zähne haben eine Länge von $15-20 \mathrm{~cm}$; sie wurden während einer Nacht (12./13. XI. 1971) gebildet, als ein ,Norte'-Einbruch den Vulkangipfel in Nebel hüllte, ansonsten aber keine Niederschläge $\mathrm{zu}$ verzeichnen waren. Aufnahme: Heine, 13. XI. 1971.

Ice edging on the angular block rubble of the Malinche Peak at an altitude of about $4450 \mathrm{~m}$. The ice teeth are about $15-20 \mathrm{~cm}$. long; they formed during the course of one night (12/13. xi. 1971) when a "Norte" broke in, shrouding the peak of the volcano in mist, but no other precipitation was recorded. 


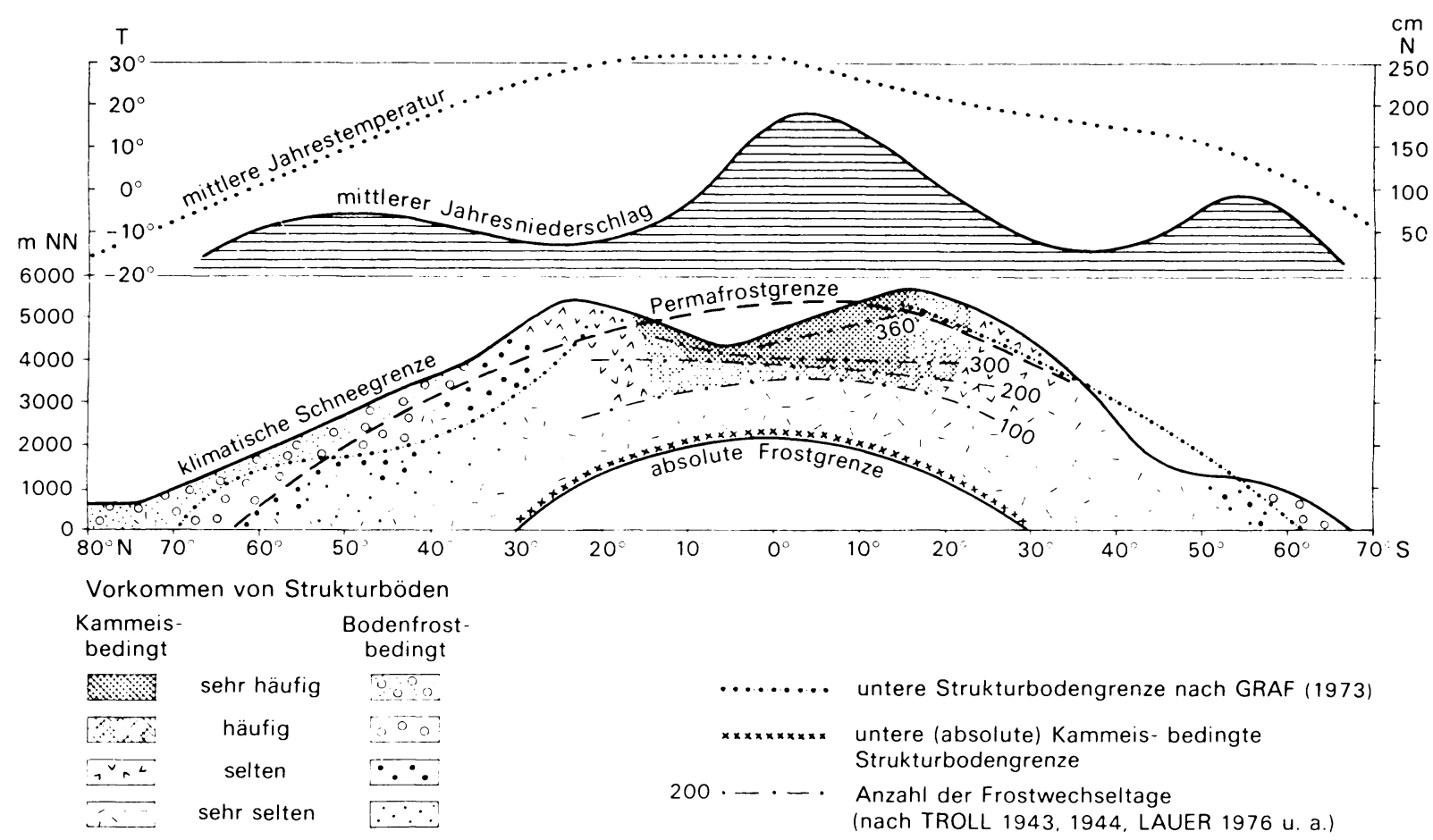

$A b b$. 2: Schematische Darstellung der horizontalen und vertikalen Verbreitung der Frostmusterböden

Schematic presentation of the horizontal and vertical distribution of frost patterned soils

tropen und Tropen eiszeitliche und rezente Strukturbodengrenzen gegenübergestellt, so werden heute oft zwei auf verschiedene Prozesse zurückgehende Formengruppen verglichen, sofern nicht ausdrücklich nur bodenfrostbedingte rezente Formen herangezogen werden. Die Ergebnisse derartiger Vergleiche sind daher stets problematisch, zumal häufig nicht einmal das Alter der fossilen Frostmusterböden genau bekannt ist, woraus sich weitere Probleme ergeben, die im Zusammenhang mit der pleistozänen Schneegrenzrekonstruktion diskutiert werden sollen.

\section{Eiszeitliche Schneegrenzen}

Uber eiszeitliche Schneegrenzdepressionen in den Subtropen und Tropen liegen zahlreiche Berichte vor. Uber die genaue Datierung der Vergletscherungsspuren, die zur Rekonstruktion der eiszeitlichen Schneegrenzen führten, gibt es so gut wie gar keine Mitteilungen. Aus den wenigen Arbeiten jedoch, die absolute Altersangaben - zumeist ${ }^{14} \mathrm{C}$-Datierungen - nennen, ist ersichtlich, daß die Annahme, die Hauptgletschervorstöße fielen in den Subtropen und Tropen mit den Hochständen der Inland- und Gebirgsvergletscherungen der gemäßigten Zonen zeitlich zusammen, überpruift werden muß. Ich möchte dazu einige Beispiele anführen.

(1) In M exiko sind die Voraussetzungen für eine genaue Datierung der Vergletscherungen günstig.
An vielen Vulkanen wurden im Jungquartär Moränen, jungquartäre Böden, äolische, kolluviale, solifluidale und fluviale Sedimente im Wechsel mit vulkanischen Locker- und Festgesteinen gebildet bzw. abgelagert. Tephra-Schichten enthalten häufig Vegetationsreste, so daß ${ }^{14} \mathrm{C}$-Datierungen vorgenommen werden können. Eine Chronostratigraphie konnte erarbeitet werden, in der die einzelnen jungquartären Vergletscherungen und deren Ablagerungen eingeordnet werden können. Das Alter, mitunter auch die Dauer eines Gletschervorstoßes lassen sich bestimmen (Heine 1975 b, 1976 a). Chronostratigraphien für verschiedene Vulkangebirge (Sra. Nevada mit Popocatépetl und Iztaccíhuatl, Malinche, Nevado de Toluca) ergeben übereinstimmende Ergebnisse. Abb. 3 zeigt die Schneegrenzdepressionen im Jungquartär für Mexiko (vgl. Heine 1976 a). Während des Maximums der letzten Kaltzeit (ca. 21000-13500 BP) gab es in Mexiko keine starken Vergletscherungen. Die eiszeitlichen Schneegrenzrekonstruktionen von WiLHELMY (1957), White (1962), Hastenrath (1968) und HeuBERGER (1974) sind daher für den Bereich der trockenen mexikanischen Randtropen nicht zutreffend, denn einerseits gibt es für trockene letzteiszeitliche Verhältnisse, die größere Vergletscherungen ausschließen, im mexikanischen Raum viele Hinweise (SEars \& Clisby 1955; Heine 1975 b; Klaus 1973) und andererseits zeichnet sich die Pleistozän/Holozän-Grenze klimatisch wie vegetationsgeschichtlich erst nach $9000 \mathrm{BP}$ 


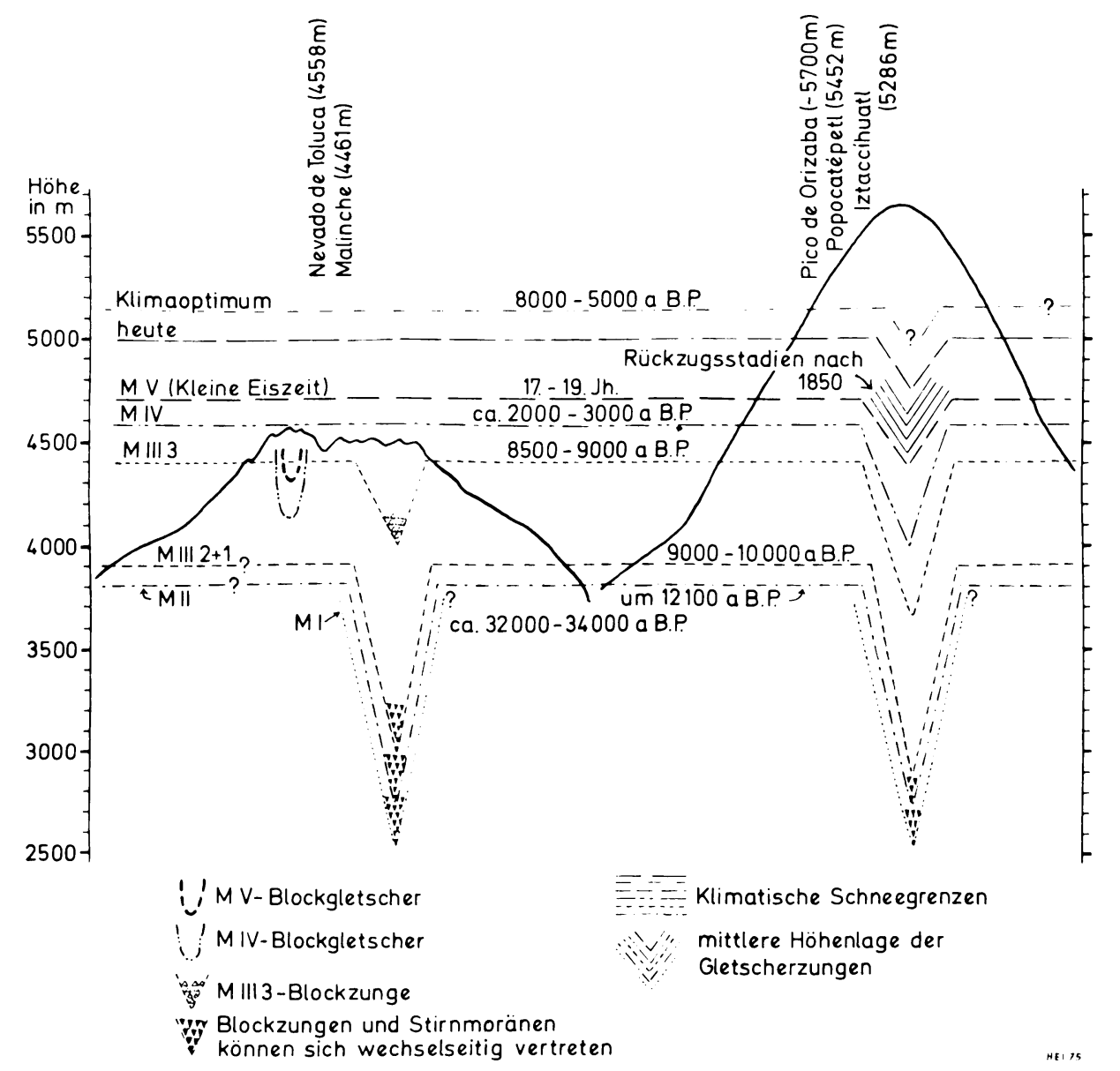

$A b b$. 3: Rekonstruktion klimatischer Schneegrenzen für verschiedene Vergletscherungen des Jungquartärs für Zentralmexiko Reconstruction of climatic snow lines for several different glaciations during the early Quaternary in Central Mexico

ab (Heine \& Ohngemach 1976; Beard 1973). Die maximale Vergletscherung der letzten Eiszeit i. e. S. (ca. 22000-10000 BP) fällt in Mexiko in die Zeit zwischen 12500 und $9000 \mathrm{BP}$.

(2) Venezuela. Exakte Angaben über Gletschervorstöße im Andenraum des tropischen Südamerika sind selten und zudem nicht immer eindeutig in ihrer Aussage. Aus Venezuela nennt Schubert (1972, $1974 \mathrm{a}+\mathrm{b}, \quad 1975)$ einen spätwisconsinzeitlichen Hauptgletschervorstoß, der von einem jüngsten (letzten) Wisconsin-Gletschervorstoß gefolgt wurde, der die Hauptendmoränen in $3000-3500$ m NN bildete. Das Alter der Hauptendmoränen wird auf ca. 11000 bis $10000 \mathrm{BP}$ geschätzt und das ältere Moränenstadium wird allein deshalb mit dem Wisconsinmaximum parallelisiert, weil „keine Moränen in tieferer Lage gefunden wurden".

(3) Aus P e r u gibt Clapperton (1972) Angaben für verschiedene Gletschervorstöße, die vermutlich alle in das Spätwisconsin und Holozän fallen. Wegen fehlender älterer Moränen vermutet Clapperton, daß die Anden Perus im Pleistozän noch nicht die Höhe hatten, um über die stark abgesenkte Schneegrenze (700 m nach HASTENRATH, 1971; 1500-2000 m allein für das Spätwisconsin nach Clapperton, 1972) hinauszuragen. - Aus dem Ayacucho-Tal - zwischen Lima und Cuzco in Peru gelegen - nennt MacNeish (1971) verschiedene spätquartäre Klimaschwankungen thermischer und hygrischer Art, die mit möglichen Vergletscherungsphasen korreliert werden; gleichzeitig wird schematisch hervorgehoben, daß sich die Gletschervorstöße des Ayacucho-Tales entgegengesetzt zu den nordamerikanischen Vorstößen verhielten.

(4) Chile und Argentinien. Im außertropischen Südamerika (Chile und Argentinien) zeichnet sich ein gleicher zeitlicher Ablauf der Gletschervorstöße ab wie in Nordamerika (Heusser 1973; Mercer 1972, 1973, 1976; Caviedes \& Paskoff 1975) (Abb. 4). Im winterfeucht-sommertrockenen Chile zwischen 29 und $34^{\circ} \mathrm{S}$ ist ebenfalls mit der 


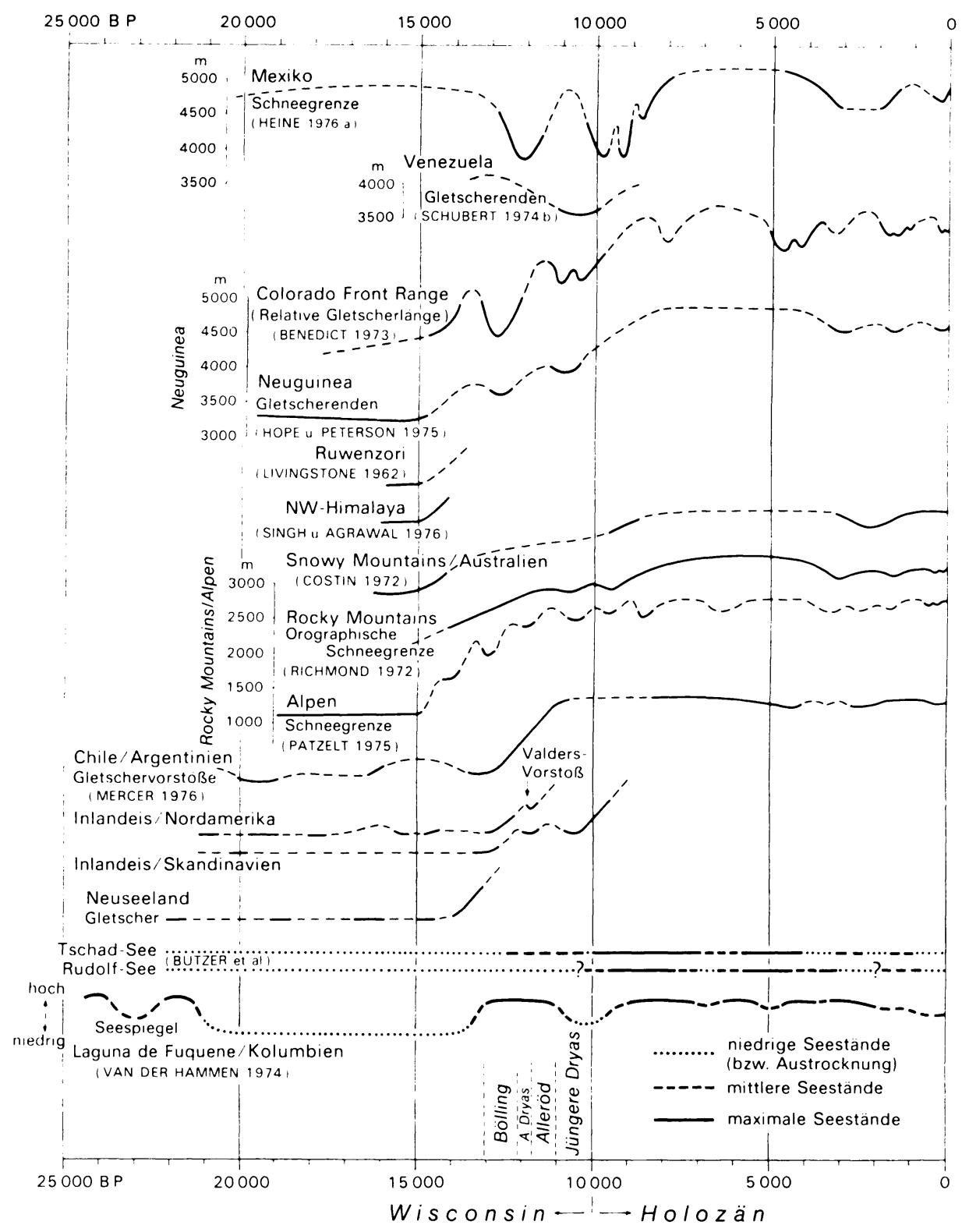

Abb.4: Schwankungen von Schneegrenzen, Gletscherenden und Seespiegelständen für ausgewählte Gebiete während des Spätglazials und Holozäns

Fluctuations of snow lines, glacier ends and lake levels in selected areas during the late glacial and holocene periods

Maximalvergletscherung während des Maximums der letzten Eiszeit zu rechnen (CAviedes \& PASKoff 1975; MERCER 1976).

(5) Norda me rika. Es fehlt nicht an Korrelationsversuchen der verschiedenen Vergletscherungsphasen Nordamerikas (vgl. Birkeland et al. 1971; MERCER 1976; HeINe 1975 b). Hier sollen lediglich einige neuere, vor allem durch eine absolute Datierung abgesicherte Ergebnisse genannt werden. Mercer (1976) gibt einen zusammenfassenden, auf neuesten Daten beruhenden vergleichenden Überblick. BENEDICT (1973,
1975) zeichnet ein Bild der Verhältnisse aus der Colorado Front Range, Richmond (1972) für das Yellowstone-National-Parkgebiet (vgl. Abb. 4). Wisconsinzeitlich sind die zwischen 17000 und 12000 BP datierten terrigenen Sedimente vor der südkalifornischen Küste, die einen größeren Abfluß und höhere Sedimentfracht vom Land ins Meer bezeugen, d.h. pluviale Bedingungen während des Wisconsinmaximums (Gorsline \& Prensky 1975).

(6) A f rika. Mitteilungen aus dem tropischen Afrika über Datierungen von Gletschervorstößen lie- 
gen nicht vor; nur vom Ruwenzori ist bekannt, daß das Gletscherabschmelzen der maximalen letzteiszeitlichen Vergletscherung bereits vor über $14700 \mathrm{BP}$ begonnen hat (Livingstone 1962; Heine 1974; MerCER 1976). Die Befunde über thermische und hygrische Klimaschwankungen aus vielen anderen Beobachtungen zeigen jedoch auch für Afrika ein sehr differenziertes Bild (HEINE 1974), weshalb man dort nicht verschiedene Moränen a priori als altersgleich ansprechen darf.

(7) $\mathrm{Himalaya.} \mathrm{Ein} \mathrm{Zurückweichen} \mathrm{der} \mathrm{Glet-}$ scher im nordwestlichen Himalaya wird von SINGH \& AGRAWAL (1976) schon ab 14000-15000 BP nachgewiesen.

(8) $\mathrm{Neuguin}$ e a. Die maximale Vergletscherung hat in Neuguinea ebenfalls wie am Ruwenzori und im NW-Himalaya vor über $14000 \mathrm{BP}$, d. h. während des Wisconsinmaximums, gelegen (GALlowAY et al. 1973; Hope \& Peterson 1975; Bowler et al. 1976).

(9) A u stralie n. Costin (1972; vgl. Bowler 1975, 1976; Bowler et al. 1976) nennt für die Snowy Mountains die Zeit um 15000-14000 BP in Verbindung mit bereits abgeschmolzenen letzteiszeitlichen Gletschern. Als Minimumalter des endgültigen Eisabschmelzens werden ${ }^{14} \mathrm{C}$-Alter um $9000 \mathrm{BP}$ angegeben (Abb. 4).

Die bereits vorliegenden, oben zitierten spärlichen Angaben lassen erkennen, wie fragwürdig Rekonstruktionen der letzteiszeitlichen Schneegrenzdepression sein müssen, da sicherlich oftmals nicht zeitgleiche Bildungen miteinander korreliert und interpretiert werden. Zudem werden oft Vermutungen geäußert, die allein aus der Annahme resultieren, die Gletscherspuren in tropischen und subtropischen wie außertropischen Gebieten seien altersgleiche Bildungen. Als Beispiel sei auf die postulierte junge Hebung der Anden wegen der in Peru fehlenden pleistozänen Moränen verwiesen; hier zeigt sich eine Lösung des Problems, wenn man - wie in Mexiko - nicht von einer hochglazialen wisconsinzeitlichen Maximalvergletscherung ausgeht, sondern die großen Gletschervorstöße (wie ja auch bei verschiedenen Autoren nachzulesen ist) in das Spätglazial/Frühholozän datiert. $\mathrm{Da} ß$ es in den Anden auch ältere Vergletscherungen gegeben hat, zeigt - neben vielen älteren Beobachtungen - die absolute Datierung einer Moräne in den kolumbianischen Anden auf über 100000 BP durch Herd \& NaEser (1974); auch die pleistozäne Vegetationsgeschichte der Nordanden (vgl. vaN DER HAMMEN 1974) stellt die allzu junge Heraushebung der Anden in Frage. Die tektonischen Erklärungen der voreilig datierten morphologischen Beobachtungen erübrigen sich nämlich, wenn man von einem differenzierten zeitlichen Ablauf der Schneegrenzdepressionen ausgeht. KновZI \& UsSELMANN (1973) belegen für Kolumbien ein trockenes Glazialklima und haben das Problem, diese Erkenntnis mit den feuchten Verglet- scherungsphasen in Einklang zu bringen; die Annahme einer spätwisconsinzeitlichen Vergletscherung nach dem relativ trockenen Glazial i. e. S. weist auch hier auf eine Lösung des Problems.

Sehr problematisch sind aufgrund des Gesagten Rekonstruktionen des Würm/Wisconsin-Klimas u. a. anhand der oft sicherlich nicht altersgleichen Moränen bzw. der Schneegrenzdepressionen, wie sie z. B. von Hastenrath (1971) und Suzuki (1973) für Südamerika und von BARRY \& Williams (1975) für die Südhemisphäre vorgenommen wurden.

\section{Folgerungen}

Für die letzte Eiszeit lassen sich anhand der relativ detaillierten Vergletscherungsabläufe mehrere Schneegrenzdepressionen in den Subtropen und Tropen nachweisen, die jedoch nicht synchron verliefen (Abb. 4 und 5). Folgender Trend läßt sich erkennen: In den immerfeuchten Tropen, die auch während des Maximums der letzten Kaltzeit im Bereich der innertropischen Zone i.S. FAIRbridges (1972) lagen, und den gemäßigten Breiten sowie den winterfeucht-sommertrockenen subtropischen (Mediterran-)Gebieten sind Gletschervorstöße alpinen Typs gleichzeitig und in enger Verbindung mit Temperaturschwankungen erfolgt; die maximale Vergletscherung fällt in das Wisconsin-Hochglazial. Auch die beiden großen Inlandeise, das nordamerikanische und skandinavische, fügen sich im wesentlichen in dieses Schema ein, wenn auch bisher keine befriedigende Korrelierung der einzelnen Eisvorstöße erzielt werden konnte. Das mag aber in lokalen Ursachen zu suchen sein. So muß man sicherlich einzelne Eisvorstöße als sogenannte Eisausbrüche (surges) deuten, wie dies Emiliani et al. (1975) beispielsweise für den Valders-Vorstoß des Michigan-See-Lobus tun, der sich nicht mit anderen Beobachtungen korrelieren läßt. Auch scheint das Verhalten der Gletscher in weit voneinander entfernten Gebieten (z. B. Chile und Alpenraum) im einzelnen durch lokale Faktoren unterschiedlich $\mathrm{zu}$ sein (Abb. 4). Allein aus dem Unterschied der letzteiszeitlichen Temperaturerniedrigung von ca. $10-12^{\circ} \mathrm{C}$ auf der Nordhemisphäre und von nur ca. 4-6 ${ }^{\circ} \mathrm{C}$ auf der Südhemisphäre (SERgiN \& CHIzhov 1976) müssen sich in der Zeit des weltweiten Temperaturanstiegs (ca. 15000-8000 BP) unterschiedliche Bedingungen für kleinere Gletscherschwankungen ergeben (vgl. Kap. V).

Im Bereich der wechselfeuchten Tropenklimate wie auch der tropischen Trocken-Halbwüstenklimate (vgl. Troll \& PAFFEN 1964) zeichnen sich mehrere kräftige Gletschervorstöße erst am Ende der letzten Eiszeit $a b$, als die Temperaturen zwar noch kühler als heute, die Niederschläge aber zeitweilig recht hoch waren. Leider liegen aus diesen Gebieten nur einige wenige detaillierte Chronostratigraphien vor, die Vergletscherungen genau erfassen. Das Bild läßt sich aber 

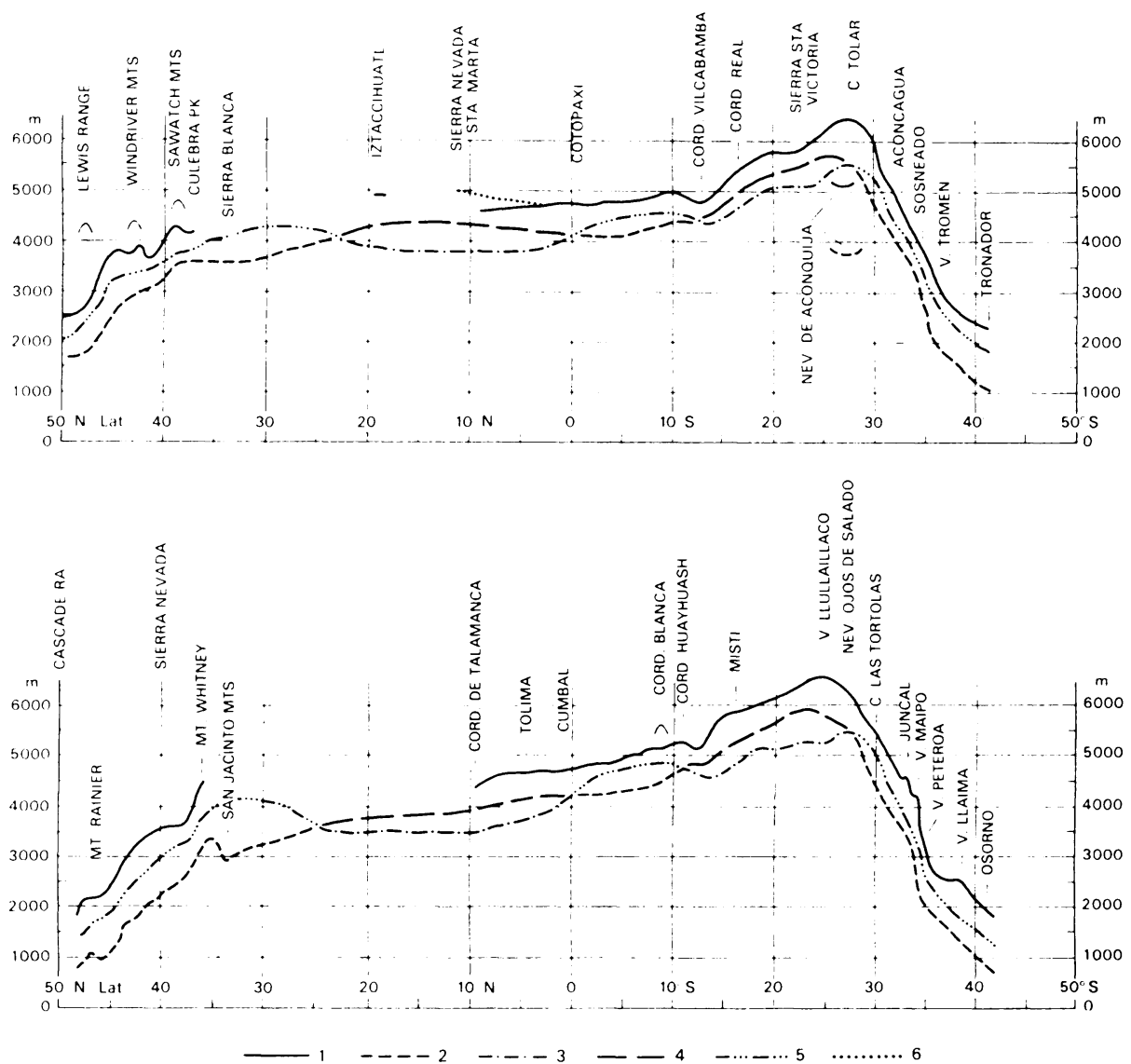

Abb. 5: Hochglaziale, spätglaziale und rezente Schneegrenze in den östlichen Anden und den Rocky Mountains (oben) und den westlichen Anden und den Küstengebieten Nordamerikas (unten). Der Verlauf der rezenten Schneegrenze und die tiefste Lage der pleistozänen Schneegrenzen sind von Heuberger (1974) übernommen und geringfügig korrigiert.

1 Rezente Schneegrenze; 2 tiefste Lage der pleistozänen (Wisconsin) Schneegrenze mit hochglazialem Alter (ca. 20000 bis $15000 \mathrm{BP}$ ); 3 tiefste Lage der pleistozänen (Wisconsin) Schneegrenze mit spätglazialem Alter (ca. 13 000-9000 BP); 4 hochglaziale letzteiszeitliche Schneegrenze oberhalb der späteiszeitlichen Maximal-Vergletscherung; 5 spätglaziale letzteiszeitliche Schneegrenze oberhalb der hochglazialen Maximal-Vergletscherung; 6 rezente Schneegrenze der Zentralcordillere

High glacial, late glacial and recent snow line in the eastern Andes and the Rocky Mountains (above), and the western Andes and the coastal mountains of North America (below). The course of the recent snow line and the lowest position of the Pleistocene snow lines are taken from Heuberger (1974), with slight corrections.

1 Recent snow line; 2 Lowest position of Pleistocene (Wisconsin) snow line of high glacial age (ca. 20,000-15,000 B.P.); 3 Lowest position of Pleistocene (Wisconsin) snow line of late glacial age (ca. 13,000-9,000 B.P.); 4 High glacial snow line of the last Ice Age snow line above the late Ice Age maximum glaciation; 5 Late glacial snow line of the last Ice Age above the high glacial maximum glaciation; 6 Recent snow line of the Central Cordilleras

vervollständigen, wenn andere paläoklimatische Beobachtungen hinzugezogen werden.

Für Mexiko konnte an verschiedenen Stellen aufgezeigt werden, daß das Hochglazial von trocken-kalten Klimaverhältnissen geprägt wurde (zuletzt HeINE 1975 b). Für Kuba nennen SHANzer et al. (1975) aride und kühle Bedingungen während der letzteiszeitlichen Meeresregression (= Hochglazial) und humide und warme Verhältnisse bei Transgressionen; für den westlichen Golf von Mexiko werden anti- zyklonal bestimmte Strömungsverhältnisse für das Hochglazial belegt (BrunNer \& CoOley 1976); das Klima der Galapagos-Inseln wird als arid zwischen 34000 und 10000 BP bezeichnet (HouvenaGHEL 1974); für das tropische Südamerika wurde bereits eine Zusammenstellung gegeben (Heine 1974); sie kann ergänzt werden durch die neueren Ergebnisse von VAN DER HAMMEN (1974) und VAN GEel \& VAN DER HAMMEN (1973), aus denen hervorgeht, daß im Bereich der kolumbianischen Anden und dem nörd- 
lichen tropischen Südamerika zwischen 21000 und $13000 \mathrm{BP}$ aridere Verhältnisse geherrscht haben und daß im Spätglazial und Holozän mehrere hygrische Klimaschwankungen auftraten. Während WarReN (1970) für den Sudan $\left(10-18^{\circ} N\right)$ für die Zeit zwischen 21000 und 9000 BP noch humidere Bedingungen vermutet, obgleich er auch die mögliche Korrelierung der humiden Phase mit einer zwischen 11000 und 8000 BP datierten Periode des Weißen Nil-Sees nennt, ergeben sich aus den gesammelten ${ }^{14} \mathrm{C}$-Daten für das innertropische Afrika (STREet \& Grove 1976; Abb. 6) recht gute Ubereinstimmungen zu den Befunden aus dem tropischen Amerika. Daß die zentrale Sahara zwischen 19000 und 13000 BP aride Klimaverhältnisse aufwies, ergibt sich u.a. auch aus den Regenerationsmöglichkeiten des Grundwassers (KLITZSCH et al. 1976); die Alter der untersuchten Grundwässer stimmen mit den von GEYH \& JäKEL (1974) statistisch ermittelten ariden und humiden Phasen der Sahara überein. Diese Ergebnisse bestätigen die früheren Schlußfolgerungen von mir (HEINE 1974) (vgl. Abb. 4). Auch aufgrund biogeographischer Forschungen wird für das Wisconsin-Maximum eine aridere Zeit für das tropische Südamerika angenommen (Müller 1974). Wenn Schubert $(1974 \mathrm{a}+\mathrm{b}, 1975)$ einerseits die Hauptendmoränenwälle der venezolanischen Anden auf ca. 11000-10000 BP datieren kann und andererseits aus Mexiko ebenfalls spätwisconsinzeitliche
Hauptendmoränen genannt werden (HeINE 1975 b), dann ist man geneigt, die Hauptendmoränen in den Cuchumatanes (Guatemala) (Hastenrath 1974 a) und in der Cordillera de Talamanca (Costa Rica) (Weyl 1956; Hastenrath 1973 b) nicht ohne ausreichende Beweise in das Wisconsin-Hochglazial zu stellen, wie es bisher von den Bearbeitern mit Vorbehalt getan wurde, zumal viele neue Befunde aus dem mittelamerikanischen Raum und dem angrenzenden nordwestlichen Südamerika auf eine mögliche chronostratigraphische Einordnung der genannten Hauptendmoränen in das ausgehende Wisconsin wejsen. Schneegrenzdepressionen, die für den Gesamtbereich der N-S-verlaufenden amerikanischen Kettengebirgsgürtel rekonstruiert werden, müssen daher den Faktor ,Zeit' unbedingt berücksichtigen (Abb. 5). Gleiches gilt auch für die jungquartären Schneegrenzrekonstruktionen in Afrika, Asien und Australien (vgl. u. a. für Australien, Tasmanien und Neuguinea: BOwler et al. 1976).

\section{Ausblick}

Abschließend soll der Versuch unternommen werden (Abb. 7, Beil. VII), die derzeitigen relevanten Befunde hypothetisch zu einem Gesamtbild zusammenzufassen. Während die Befunde über die Vergletscherungen und die Aridität im Hochglazial auf neueren

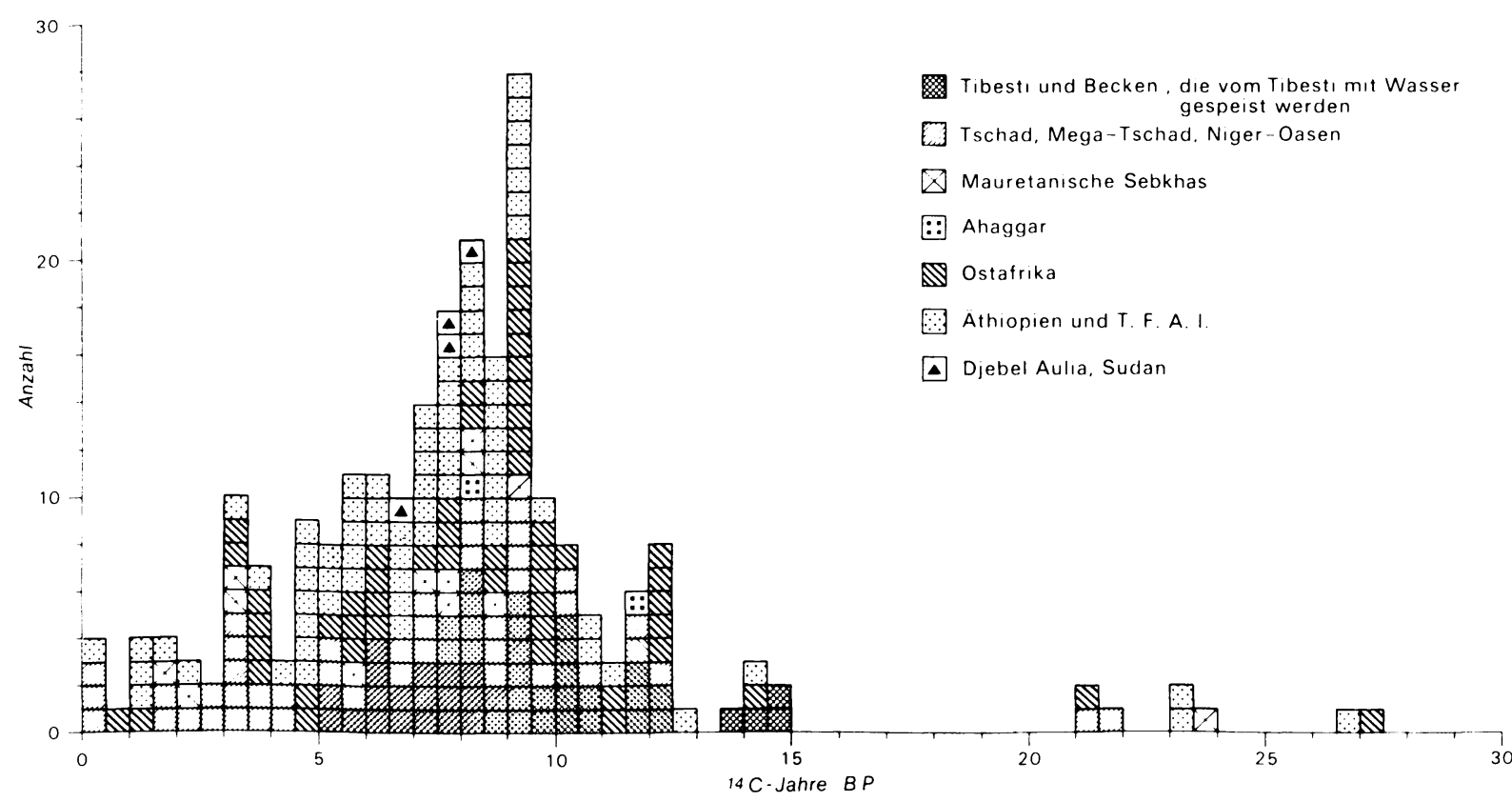

Abb. 6: Histogramm von $238{ }^{14} \mathrm{C}-\mathrm{Daten}$ aus dem tropischen Afrika, die hohe und mittlere Scespiegelstände anzcigen. Lakustrische Sedimente (= pluviale Phasen) vor $21000 \mathrm{BP}$ sind unterrepräsentiert, da die Sedimente erodiert oder unter jüngeren Ablagerungen begraben sind. Nach Street \& Grove 1976

Histogram of $238 \mathrm{C}^{14}$ data from tropical Africa, indicating high and medium lake levels. Lacustrine sediments (= pluvial phases) before 21,000 B.P. are under-represented since the sediments are croded or buried under more recent deposits. (after STREET \& Grove, 1976) 
Untersuchungsergebnissen fußen, sind die Zonen mit unterschiedlichen Zeiten der Maximal-Vergletscherungen teilweise noch sehr spekulativ, so z. B. in Zentralamerika und in Teilen der südamerikanischen Anden, ebenso in Ostafrika und Äthiopien; aus Südost-Asien liegen keine Angaben über Datierungen von Vergletscherungen vor.

Relativ gut wissenschaftlich erarbeitet sind die amerikanischen Gebiete hinsichtlich absoluter Datierungen der Vergletscherungen. Daher läßt sich der Ablauf der Vergletscherungen in den nordamerikanischen Rocky Mountains mit Chile im wesentlichen korrelieren. Die mexikanische und venezolanische Maximalvergletscherung wird ebenfalls erfaßt. Nicht geklärt ist die zeitliche Stellung der Hauptgletschervorstöße in den Anden Ecuadors und Nordperus. Hier nämlich könnte - analog zu den Verhältnissen am Ruwenzori und in Neuguinea - eine Maximalvergletscherung auch vor ca. $14000 \mathrm{BP}$ vermutet werden, dann nämlich, wenn im Hochglazial die Niederschläge zwar vermindert waren, diese jedoch eine große Vergletscherung infolge der Temperaturerniedrigung noch zuließen.

Der scheinbar problematische Verlauf der rezenten und pleistozänen Schneegrenzen im Andenbereich Südamerikas (Abb. 5) zwischen $13^{\circ}$ und $29^{\circ} \mathrm{S}$ beide Grenzen divergieren hier - war wiederholt Anlaß zu Spekulationen (Heuberger 1974); WILHELMY (1957) glaubt, daß diese "Anomalie“ auf eine Abschwächung der Trockenklimate während der letzten Eiszeit zurückzuführen ist; Suzuki (1971) schließt aus der "Anomalie" auf eine nordwärtige Verlagerung der außertropischen Westwindzone; HEUBERGER (1974) macht darauf aufmerksam, daß die Kulmination der eiszeitlichen Schneegrenze verglichen mit derjenigen von heute jedoch nicht nordwärts verschoben ist, was sich zweifellos aus einer eiszeitlichen Verlagerung der Westerlies ergeben müßte; in den Westanden ist die eiszeitliche Kulmination sogar nach Süden (zwischen $25^{\circ}$ und $29^{\circ} \mathrm{S}$ ) verlagert (HEUBERGER 1974).

Unter der Annahme, daß verschieden alte Moränen die jungpleistozäne Maximalvergletscherung der Anden anzeigen, lassen sich eventuell die strittigen Fragen lösen, dann nämlich, wenn die "pleistozäne“ Schneegrenze aus mehreren Schneegrenzen verschiedenen Alters rekonstruiert wird (Abb. 5). Die Kulmination der "pleistozänen" Schneegrenze, die aus der Uberschneidung mehrerer Schneegrenzen resultiert, zeigt nur scheinbar eine Kulmination an; sie liegt in dem Bereich der Anden, der weder im Hochglazial noch im Spätglazial besonders starke Niederschläge erhielt; die hochglazialen ariden Bereiche jedoch waren möglicherweise äquatorwärts verschoben, wie das für den neuweltlichen nordhemisphärischen Trockengürtel nachgewiesen werden konnte. Ähnliche Verhältnisse sind aufgrund biogeographischer Beobachtungen für die zentrale Namib in Südwestafrika (vAN ZINDEREN
BAKKer 1975) bzw. für die nordwestafrikanische Küste bei $18^{\circ}$ bis $20^{\circ} \mathrm{N}$ (Diester-HaAs 1976; Einsele \& Herm 1976) zu vermuten. Vielleicht darf man auch unter diesen Gesichtspunkten morphologische Befunde aus Chile interpretieren; dort läßt sich bei $29^{\circ} \mathrm{S}$ die Grenze zwischen dem Bereich mit Glatthang-Bildung und -Erhaltung im Süden und der endorëischen Zone mit Talverschüttung und GlacisBildung als dominierende Formungsmerkmale im Norden festlegen (WEISCHET 1969).

Aufgrund der bisher vorliegenden Erkenntnisse muß auch für Afrika eine stärkere raum-zeitliche Differenzierung der jungquartären Temperatur- und vor allem der Niederschlagsentwicklung (STREET \& Grove 1976) vorgenommen werden. Unter der Annahme, daß bedeutende Temperaturschwankungen auf der Erde überall \pm gleichzeitig auftraten (vgl. HEINE 1974), gewinnen die hygrischen Schwankungen besondere Bedeutung. Eine pleistozäne Absenkung der Schneegrenze, die zeitlich mit dem außertropischen Hochglazial i.e.S. zusammenfiel, scheint für NWAfrika belegt zu sein; sie fällt mit Klimaverhältnissen in NW-Afrika zusammen, die einerseits als pluvial (Diester-HaAs 1976), andererseits als recht trocken (RoHDEnburg \& SAbELberg 1973; Bos 1971; BRUNNACKER 1973; EINSEle \& Herm 1976) bezeichnet werden. Obgleich es in jüngerer Zeit vermehrte Anzeichen dafür gibt, daß auch im Mittelmeerbereich während des letzten Glazials aridere Klimabedingungen geherrscht haben, sollte man auch dort eine stärkere raum-zeitliche Unterscheidung treffen. So können im nördlichen Mediterrangebiet infolge kalter und relativ trockener Verhältnisse zwar in tieferen Lagen aridere Bedingungen auftreten, in den Gebirgen jedoch kann die starke Temperaturabsenkung Gletschervorstöße bewirken. Auch für den Vorderen Orient mag die Reliefgestaltung für differenzierte Klimaänderungen hygrischer Art verantwortlich sein. Nordwest-Syrien und das nördliche Küstengebiet des Libanon waren vermutlich relativ arid während des Hochglazials, während für das Damaskus-Becken (KAISER et al. 1973) gleichzeitig pluviale Verhältnisse belegt werden und im Gegensatz dazu für das Tote Meer ein Austrocknen des pluvialen Lisan-Sees ab ca. 20000 BP angenommen wird (HUCKRIEDE 1972; FARRAND 1973). Arides Klima herrschte im Hochglazial auch im Roten Meer und im Golf von Aden (Deuser et al. 1976). Gehen wir davon aus, daß kalte und trockene Luftmassen im Hochglazial (ca. 20000 bis 13000 BP) von Mitteleuropa bis ins nördliche Mittelmeergebiet reichten und daß die pluvialen Bedingungen im östlichen Mittelmeergebiet von den aus Westen heranziehenden Zyklonen bei recht kühlen Temperaturen verursacht wurden, die für die Küste der Levante und die Gebirge mehr Niederschläge brachten als für die im Regenschatten liegenden Grabengebiete um das Tote Meer, dann erscheinen die Widersprüche der paläoklimatischen Befunde aus dem Vorderen Orient 

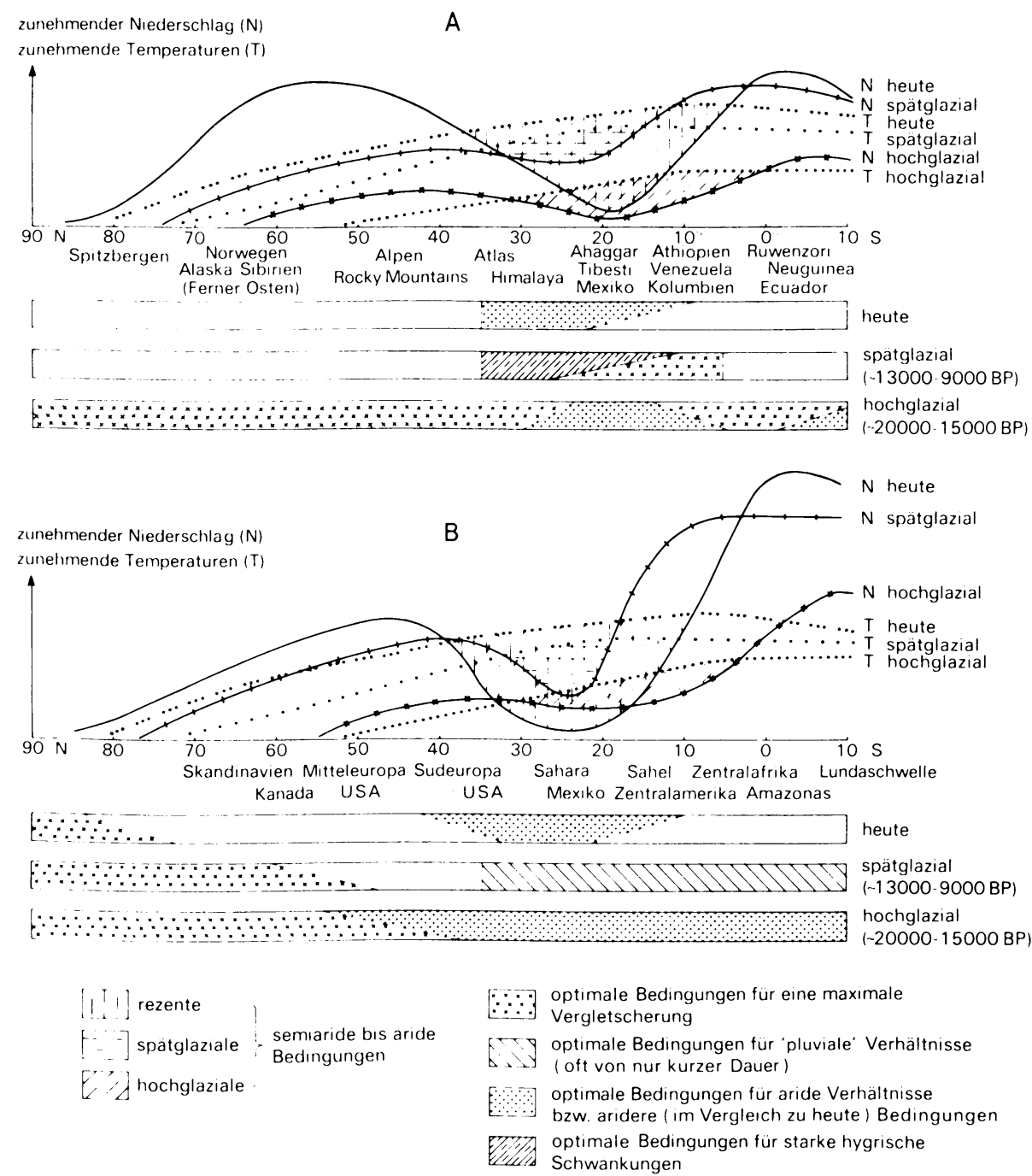

Abb. 8: Versuch einer schematischen Darstellung humider und arider Klimabedingungen zwischen Nordpol und Aquator für das letzteiszeitliche Hochglazial (ca. 20000-15000 BP), das Spätglazial (ca. 13 000-9000 BP) und die Gegenwart für Gebirge (A) und Tiefländer (B). Der Hauptabschnitt der letzten Kaltzeit zeigt eine maximale Temperaturdepression bei allgemein reduzierten Niederschlägen. Am Ausgang der letzten Kaltzeit bringen vor allem hygrische Klimaschwankungen - synchron (?) oder zeitlich entgegengesetzt (?) - pluviale Phasen für die meisten Gebiete der heutigen ariden und wechselfeuchten Tropen und Subtropen. Pluviale Erscheinungen werden z.T. insbesondere als Fernwirkungen der Vorgänge vorgetäuscht, die sich in Bergländern bei herabgedrückten, relativ humideren Höhenstufen abspielten (vgl. BRUNNACKER 1973)

Attempt at a schematic presentation of humid and arid climatic conditions between the North Pole and Equator at the high glacial stage of the last Ice Age (c. 20,000-15,000 B.P.), the late glacial period (c. 13,000-9,000 B.P.) and the present for mountains (A) and lowlands (B). The main section of the last glacial period shows a maximum temperature depression, together with an overall reduction in precipitation. Towards the end of the last glacial epoch predominantly hygric climatic fluctuations produce - synchronically? or periodically opposed? - pluvial phases for most areas of the at present arid and savanna climates of the tropics and sub-tropics. In parts pluvial phenomena are simulated, particularly as long range effects of those processes which took place in mountainous uplands, under depressed, relatively more humid altitudes (cf. BRUNNACKER, 1973)

nicht mehr so gravierend (vgl. Abb. 8). Auch ist daran zu denken, daß die größeren Gebirge der zentralen Sahara (Ahaggar- und Tibesti-Massiv) ebenfalls im Hochglazial i. c. S. von den außertropischen Zyklonen Niederschläge empfingen, während die tiefer gelegenen
Gebiete ringsum noch aride Verhältnisse aufwiesen. Eine Absenkung der Schneegrenze (Messerli 1973) bzw. der Untergrenze der Nivationsformen wäre dann dort \pm synchron zu den außertropischen Maximalvergletscherungen erfolgt (vgl. dazu BUTZER 1973; 
Williams 1976); das mag auch noch für manche Gebiete von Äthiopien zutreffen, so z. B. für das Hochland von Semien, während die weiter östlich gelegene Afar-Depression nachweislich im Hochglazial (23000 bis $11000 \mathrm{BP}$ ) ein trockenes Klima aufwies (GASSE \& RogNon 1973). Für das Rift Valley in Südäthiopien ist eine pluviale Phase erst im Spätpleistozän/Frühholozän belegt (Grove et al. 1975). Nivationsnischen, wie sie aus den Gebirgen der zentralen Sahara von Messerli (1972) beschrieben werden, sind mir aus Mexiko bekannt (Foto 5), wo sie holozänes Alter haben (vgl. Heine 1976 b) und sehr wahrscheinlich gebildet wurden, als in Zeiten häufigerer Norte-Wetterlagen vermehrte Nebelfröste an den mexikanischen Vulkanen bedeutenden Eisbesatz an den Schutthaldenblöcken erzeugten (Foto 6); das wiederum kann die Morphodynamik in der subnivalen Stufe stark beeinflussen, ohne daß dadurch jedoch in den umrahmenden Beckenlandschaften von vornherein pluviale Bedingungen vermutet werden müssen.

In Ostafrika scheinen die Rekonstruktionen pleistozäner Klima- und Landschaftsverhältnisse sehr schwierig zu sein. Die Schneegrenzdepression ist zwar für verschiedene Vulkangebirge in ihren Ausmaßen bekannt; über die zeitliche Einstufung der Vergletscherungen gibt es dagegen wenig exakte Hinweise. So ist zur Zeit nicht möglich, die - vorwiegend aus den Seespiegelschwankungen abgeleitete - Klimaentwicklung (Abb. 4 und 7) während der letzten 20000 Jahre auf die Vergletscherungen zu übertragen. Einerseits ist daran zu denken, daß infolge relativ trockener hochglazialer Verhältnisse (zuletzt: STREet \& Grove 1976) die maximalen Gletschervorstöße an den ostafrikanischen Vulkanen erst mit den pluvialen Phasen am Ende der letzten Eiszeit auftraten (analog zu den Verhältnissen im nördlichen Südamerika und Mittelamerika); andererseits ist nicht auszuschließen, daß ein abgeschwächter Nordost-Monsun während des hochglazialen Nordwinters bzw. der SE-Passat während des hochglazialen Nordsommers nur den hohen Vulkanen ausreichene Niederschläge für eine Maximalvergletscherung brachte, während die tiefer gelegenen Gebiete \pm aride Verhältnisse im ökologischen Sinne zeigten (vgl. vaN ZiNDEREN BAKKER 1967; Abb. 4). Immerhin fällt die Maximalvergletscherung des Ruwenzori (LIVINGSTONE 1962) in eine Zeit, in der pollenanalytisch für die Umgebung des Gebirges aridere Klimaverhältnisse im Vergleich zu heute nachgewiesen werden (HamiLton 1972).

Bevor eine Korrelierung der Vergletscherungen bzw. der pleistozänen Schneegrenzdepressionen ostafrikanischer Gebirge mit anderen afrikanischen Gebieten vorgenommen wird (vgl. Messerli 1973; HastenRATH 1974 b), sollten die chronostratigraphischen Verhältnisse der ostafrikanischen glazialen und periglazialen Spuren eindeutig bestimmt werden.

Im australasiatischen Raum (Neuguinea und Australien) läßt sich die pleistozäne Schneegrenzdepression chronologisch recht gut erfassen. Die Ergebnisse zeigen, daß auch dort nicht von einer Gleichzeitigkeit der Ereignisse ausgegangen werden darf. In Neuguinea war die maximale Vergletscherung vor ca. 15000 BP. Die Untersuchungen von Hope \& Peterson (1975), Bowler et al. (1976) und von Kershaw (1975) belegen relativ feuchte hochglaziale Klimaverhältnisse für die gebirgigen und nördlichen Gebiete von Neuguinea. In dieser Zeit herrschte in NordostAustralien ein im Vergleich zu heute trockeneres Kli$\mathrm{ma}$, das erst in der Zeit zwischen 11000 und 6000 BP von feuchteren Bedingungen abgelöst wurde (KERSHAw 1975). In Süd-Australien bestimmten zwischen ca. 17000 und $14000 \mathrm{BP}$ aride Verhältnisse das Geschehen (BowLER 1975, 1976; Bowler et al. 1976); in dieser Zeit sollen bereits die kleinen Gletscher der Snowy Mountains infolge Niederschlagmangels verschwunden sein, während die Kargletscher Tasmaniens gerade zu dieser Zeit durch regenbringende NordwestWinde wachsen konnten. Auch hier zeigt sich deutlich, daß auf relativ „kleinem“ Raum infolge differenzierter Niederschlagsverteilung (vor allem auch gegenüber heute) eine maximale Vergletscherung zu verschiedenen Zeiten auftrat. - Neuseeland hatte wie auch Tasmanien - die stärksten Vergletscherungen \pm synchron mit den Eisvorstößen der anderen außertropischen Gebirge (chilenische Anden, Alpen, Rocky Mountains etc.).

\section{$V$. Die Bedeutung der spätwisconsinzeitlichen Klimaschwankungen für die Schneegrenzdepressionen}

Der Übergang von der letzten Eiszeit zum Holozän wird durch markante Klimaschwankungen repräsenticrt (zuletzt FLOHN 1976). Der allgemeine weltweite Temperaturanstieg ist ab ca. $13000 \mathrm{BP}$ überall nachzuweisen. Er wird in manchen Gebieten durch die Altere und die Jüngere Dryaszeit (Abb. 4) unterbrochen. Diese kalten Episoden sind am kräftigsten im randlichen Bereich der großen nordhemisphärischen Inlandeise (Nordamerika-Grönland, Skandinavien) ausgeprägt, und zwar in kontinentalen Räumen wescntlich deutlicher als in maritimen atlantischen $\mathrm{Kü}$ stenregionen. In den Tropen finden sich nur palynologische Hinweise auf diese Schwankungen (VAN DER Hammen 1974; van Zinderen Bakker \& Coetzee 1972); in den ehemals vergletscherten Gebieten der Südhemisphäre schließlich fehlen eindeutige Beweise für die Altere und Jüngere Dryaszeit ganz, so in Chile (Mercer 1976), Australien (Bowler 1975, zit. nach FlOHN 1976; Bowler et. al. 1976) und Neuseeland. Viele neuere Untersuchungen - so auch die Entwicklung in den Alpen (Abb. 4) - zeigen, daß bereits $a b$ ca. $15000 \mathrm{BP}$ mit einem Anstieg der Temperaturen und einem damit verbundenen Rückgang der Gletscher in den Außertropen und den Feuchttropen gerechnet werden muß (vgl. DonN 1976). 
Die Ursachen für die heftigen Temperatur-Oszillationen der Alteren und Jüngeren Dryaszeit, die sehr plötzlich und in wenigen Jahrhunderten abliefen, kann man mit einem schnellen Anwachsen der Eismassen im nordatlantischen Raum erklären, die nach Aufbrechen des arktischen Schelfeises infolge der weltweiten Erwärmung durch die erhöhte Verdunstung und Niederschlagstätigkeit gebildet wurden; ein derartiger „lokaler" Rückfall in eiszeitliche Verhältnisse mag sich mehrmals wiederholt haben (Altere und Jüngere Dryaszeit) und würde die extremen - in tropischen und subtropischen Gebieten nur schwach oder gar nicht registrierten - Temperaturschwankungen möglicherweise erklären (MERCER 1969). Hier fügen sich auch die in Abb. 4 festgehaltenen Beobachtungen ein, aus denen hervorgeht, daß in Mexiko beispielsweise der Klimagang nicht direkt von den kurzen heftigen, für Grönland, Europa (außer Südengland) und Nordamerika nachgewiesenen Klimaschwankungen thermischer Art beeinflußt wurde; erst als Folge der Veränderungen der allgemeinen Zirkulation der Atmosphäre, verursacht durch die vergrößerten Gletscher in Europa und Nordamerika, gewinnen die thermischen Klimaschwankungen der Älteren und Jüngeren Dryaszeit auch für Mexiko an Bedeutung, wo sie sich vermutlich mehr als hygrische, denn als thermische Oszillationen auswirkten; auch für das nördliche Südamerika bringen die Kältephasen abgeschwächte Einflüsse, nämlich nur noch geringfügige Veränderungen der Vegetation und der Seestände, während die Vergletscherung der Nordanden nur noch auf die Schwankung der Jüngeren Dryaszeit zu reagieren scheint (vgl. auch die Seespiegelschwankungen der Laguna de Fuquene / Kolumbien in Abb. 4!). In Chile schließlich wird die Jüngere Dryaszeit allein palynologisch durch HeUSSER (1974) belegt, nicht aber glazialgeologisch, denn zwischen 13000 und 11000 BP erfolgt dort das Abschmelzen der Gletscher bis auf ihren heutigen Stand, ohne daß die Gletscher in der Jüngeren Dryaszeit noch einmal vorrückten, wie das beispielsweise für die Alpen belegt werden kann'2). Anbei sei noch bemerkt, daß der Valders-Vorstoß in Nordamerika nicht mehr (wie früher) der Jüngeren Dryaszeit zugeschrieben wird, sondern als Eisausbruch um $11850 \mathrm{BP}$ als Folge der in das Eis eindringenden Schmelzwässer gedeutet wird (WRIGHT 1971; EMILIANI et al. 1975).

Werden all die hier nur kurz umrissenen Differenzierungen im raum-zeitlichen Ablauf der weltweiten jungquartären Vergletscherungen berücksichtigt, so stellt sich die Frage, ob es noch sinnvoll erscheint, eine pleistozäne Schneegrenze zu rekonstruieren und diese

2) Vegetationsentwicklung und Gletscherverhalten in Südchile lassen vermuten, daß die Altere und die Jüngere Dryaszeit dort hygrisch stärker in Erscheinung traten als thermisch, denn thermische Schwankungen pflanzen sich weltweit nur relativ langsam fort (vgl. FLOHN 1974), hygrische dagegen - über die allgemeine Zirkulation der Atmosphäre und deren Veränderungen - relativ schnell. dann für weiterführende Betrachtungen zu benutzen (z. B. für Klimarekonstruktionen). Hinzu kommt der Umstand, daß in den ariden Subtropen und Tropen die Verdunstung so stark ins Gewicht fällt, daß die Schneegrenze als allgemeiner Klimaanzeiger fragwürdig wird (Grar 1975) und ebenso ihre Korrelation mit der Schneegrenze feuchterer Gebiete. Hier schlägt Graf (1975) eine neue Definition vor, nämlich die Schneegrenze in den Tropen durch die untersten Vorkommen von zweijährigem Bodenfrost (Permafrost) zu bestimmen, welche im Bereich eines Gletschernährgebietes oder in nicht vergletschertem Gebiet (aride bis extrem aride Gebirge) festgestellt werden.

\section{Literatur}

Barry, R. G. \& Williams, J.: Experiments with the NCAR Global Circulation Model using Glacial Maximum Boundary Conditions: Southern Hemisphere Results and Interhemispheric Comparison. - Quaternary Studies, ed. R. P. Suggate \& M. M. Cresswell, Roy. Soc. New Zealand, S. 57-66, Wellington 1975.

Beard, J. H.: Pleistocene/Holocene Boundary and Wisconsinian substages, Gulf of Mexico. - The Wisconsinan Stage, ed. R. F. Black et al., Mem. Geol. Soc. Amer., 136, S. 277-316, Washington 1973.

Beckmann, G. G., Thompson, C. H. \& Hubble, G. D.: Linear Gilgai. - The Australian Geographer, XII, S. 363 $-366,1973$.

Benedict, J. B.: Chronology of Cirque Glaciation, Colorado Front Range. - Quaternary Research, 3, S. 584-599, 1973.

- : Prehistoric Man and Climate: The View from Timberline. - Quaternary Studies, ed. R. P. Suggate \& M. M. Cresswell, Roy. Soc. New Zealand, S. 67-74, Wellington 1975.

- : Frost Creep and Gelifluction Features: A Review. Quaternary Research, 6, S. 55-76, 1976.

Birkeland, P. W., Crandell, D. R. \& G. M. Richmond: Status of Correlation of Quaternary Stratigraphic Units in the Western Conterminous United States. - Quaternary Research, 1, S. 208-227, 1971.

Bos, R. H. G.: Quaternary Evolution of a Mountainous Area in N.W. Tunisia - a Geomorphological and Pedological Analysis. - Publ. Fys.-Geogr. Bodenk. Lab. Univ. Amsterdam 19, S. 1-167, 1971.

Bowler, J. M.: Deglacial Events in Southern Australia: Their Age, Nature, and Palaeoclimatic Significance. -Quaternary Studies, ed. R. P. Suggate \& M. M. Cresswell, Roy. Soc. New Zealand, S. 75-82, Wellington 1975.

- : Aridity in Australia: Age, Origins and Expression in Aeolian Landforms and Sediments. - Earth-Sci. Rev., 12, S. 279-310, 1976.

Bowler, J. M., Hope, G. S., Jennings, J. N., Singh, G. \& WALKeR, D.: Late Quaternary Climates of Australia and New Guinea. - Quaternary Research, 6, S. 359-394, 1976.

Bremer, H.: Musterböden in tropisch-subtropischen Gebieten und Frostmusterböden. - Z. Geomorph. N. F., 9, S. 222-236, 1965. 
Brunner, C. A. \& Cooley, J. F.: Circulation in the Gulf of Mexico during the last glacial maximum $18000 \mathrm{yr}$ ago. - Bull. Geol. Soc. Amer., 87, S. 681-686.

BRUNNACKER, K.: Einiges über Löß-Vorkommen in Tunesien. - Eiszeitalter u. Gegenwart, 23/24, S. 89-99, 1973.

Butzer, K. W.: Past climates of the Tibesti mountains, central Sahara. - Geogr. Review, 63, S. 395-397, 1973.

Butzer, K. W., Isaac, G. L., Richardson, J. L. \& WashBourn-Kamau, C.: Radiocarbon Dating of East African Lake Levels. New observations provide fresh insights into late Quaternary paleoclimates. - Science, 175, S. $1069-1076,1972$.

Caviedes, C. N. \& Paskoff, R.: Quaternary Glaciations in the Andes of North-Central Chile. - J. Glaciology, 14 , S. $155-170,1975$.

Clapperton, C. M.: The Pleistocene Moraine Stages of West-Central Peru. - J. Glaciology, 11, S. 255-263, 1972.

Costin, A. B.: Carbon-14 Dates from the Snowy Mountains Area, Southeastern Australia, and Their Interpretation. - Quaternary Research, 2, S. 579-590, 1972.

Deuser, W. G., Ross, E. H. \& Watermann, L. S.: Glacial and pluvial periods: their relationship revealed by Pleistocene sediments of the Red Sea and Gulf of Aden. Science, 191, S. $1168-1169,1976$.

Diester-HaAs, L.: Late Quaternary Climatic Variations in Northwest Africa Deduced from East Atlantic Sediment Cores. - Quaternary Research, 6, S. 299-314, 1976.

Donn, W. L.: Comment on "Ice Age and the Thermal Equilibrium of the Earth, II," by D. P. Adam. - Quaternary Research, 6, S. 315-316, 1976.

Einsele, G. \& Herm, D.: Spätpleistozäne und holozäne Meeresspiegelschwankungen an der mauretanischen Küste und Schelf. - Abstract, 18. wiss. Tag. DEUQUA, 31.8. 4. 9. $1976,1976$.

Emiliani, C., Gartner, S., Lidz, B., Eldridge, K., Elvey, D. K., Huang, T. Ch., Stipp, J. J. \& Swanson, M. F.: Paleoclimatological Analysis of Late Quaternary Cores from the Northeastern Gulf of Mexico. - Science, 189 S. $1083-1088,1975$.

FAIRBRIDGe, R. W.: Climatology of a Glacial Cycle. Quaternary Research, 2, S. 283-302, 1972.

FARRAND, W. R.: Paleo-Environment of Prehistoric Man in the Eastern Mediterranean. - IX. INQUA Cong., Abstracts, S. 92, Christchurch/N.Z. 1973.

Flohn, H.: Background of a Geophysical Model of the Initiation of the Next Glaciation. - Quaternary Research, 4, S. 385-404, 1974.

- : Abrupt events in climatic history. - (Lecture given at the Australian Conference on Climate and Climatic Change, Melbourne, 12. Dec. 1975), Meteorol. Inst. Univ. Bonn, Nr. 59, S. 1-15, Bonn 1976.

FurRer, G. \& Freund, R.: Beobachtungen zum subnivalen Formenschatz am Kilimandjaro. - Z. Geomorph. N. F., Suppl. Bd. 16, S. 180-203, 1973.

Galloway, R. W., Hope, G. S., Löffler, E. \& Peterson, J. A.: Late Quaternary Glaciation and Periglacial Phenomena in Australia and New Guinea. - Palaeoecology of Africa, 8, S. $125-138,1973$.
Gasse, F. \& Rognon, P.: Le Quaternaire des Bassins lacustres de l'Afar. - Rev. Géogr. phys. Géol. dyn., XV, S. $405-414,1973$.

GEYH, M. A. \& JäKEL, D.: Spätpleistozäne und holozäne Klimageschichte der Sahara aufgrund zugänglicher ${ }^{14} \mathrm{C}$ Daten. - Z. Geomorph. N. F., 18, S. 82-92, 1974.

GoldthwaIt, R. P.: Frost Sorted Patterned Ground: A Review. - Quaternary Research, 6, S. 27-35, 1976.

Gorsline, D. S. \& Prensky, S. E.: Paleoclimatic Inferences for Late Pleistocene and Holocene for California Continental Borderland Basin Sediments. - Quaternary Studies, ed. R. P. Suggate \& M. M. Cresswell, Roy. Soc. New Zealand, S. 147-154, Wellington 1975.

Graf, K.: Vergleichende Betrachtungen zur Solifluktion in verschiedenen Breitenlagen. - Z. Geomorph. N. F., Suppl. Bd. 16, S. 104-154, 1973.

- : Geomorphologische Studien in den Anden und auf dem Altiplano Boliviens. (Ubersetzung) - Ztschr. Soc. Geol., 21, La Paz 1975 (im Druck).

- : Zur Mechanik von Frostmusterungsprozessen in Bolivien und Ecuador. - Z. Geomorph. N. F., 20, S. 417.447, 1976.

Grove, A. T., Street, F. A. \& Goudie, A. S.: Former lake levels and climatic change in the Rift valley of Southern Ethiopia. - Geogr. Journal, 141, S. 177-202, 1975.

Hagedorn, J.: Note on the occurence of needle ice phenomena in the southern Sinai Mountains. - Z. Geomorph. N. F., Suppl. Bd. 21, S. 35-38, 1974.

Hamilton, A. C.: The interpretation of pollen diagrams from highland Uganda. - Palaeoecology of Africa, 7, S. $45-149,1972$.

Hastenrath, S.: Certain aspects of the three-dimensional distribution of climate and vegetation belts in the mountains of Central America and southern Mexico. - Coll. Geogr., 9, S. 122-130, 1968.

- : On the Pleistocene Snow-line Depression in the Arid Regions of the South American Andes. - J. Glaciology, 10, S. 255-267, 1971.

- : Observations on the periglacial morphology of Mts. Kenya and Kilimanjaro, East Africa. - Z. Geomorph. N. F., Suppl. Bd. 16, S. 161-179, 1973 a.

- : On the Pleistocene Glaciation of the Cordillera de Talamanca, Costa Rica. - Ztschr. Gletscherkde. Glazialgeol., 9, S. 105-121, 1973 b.

- : Spuren pleistozäner Vereisung in den Altos de Cuchumatanes, Guatemala. - Eiszeitalter u. Gegenwart, 25, S. 25-34, 1974 a.

- : Glaziale und periglaziale Formbildung in Hoch-Semyen, Nord-Äthiopien. - Erdkde., 28, S. 176-186, 1974 b.

- : Weitere Beobachtungen zu Bodenfrosterscheinungen am Mt. Kenya. - Z. Gemorph. N. F., 20, S. 235-239, 1976.

Heine, K.: Bemerkungen zu neueren chronostratigraphischen Daten zum Verhältnis glazialer und pluvialer Klimabedingungen. - Erdkde., 28, S. 303-312, 1974.

- : Permafrost am Pico de Orizaba/Mexiko. - Eiszeitalter u. Gegenwart, 26, S. 212-217, 1975 a.

- : Studien zur jungquartären Glazialmorphologie mexikanischer Vulkane, mit einem Ausblick auf die Klimaentwicklung. - Das Mexiko-Projekt der DFG, VII, S. 1-178, Wiesbaden 1975 b. 
- : Schneegrenzdepressionen, Klimaentwicklung, Bodenerosion und Mensch im zentralmexikanischen Hochland im jüngeren Pleistozän und Holozän. - Z. Geomorph. N. F., Suppl. Bd. 24, S. 160-176, 1976 a.

- : Blockgletscher- und Blockzungen-Generationen am Nevado de Toluca, Mexiko. - Die Erde, 107. Jg., S. 330352, $1976 \mathrm{~b}$.

- : Zur morphologischen Bedeutung des Kammeises in der subnivalen Zone randtropischer semihumider Hochgebirge. - Z. Geomorph. N. F., 21, S. 57-78, 1977.

Heine, K. \& Ohngemach, D.: Die Pleistozän/HolozänGrenze in Mexiko. - Münster. Forsch. Geol. Paläont., 38/39, S. 229-251, 1976.

Herd, D. G. \& Naeser, C. W.: Radiometric Evidence for Pre-Wisconsin Glaciation in the Northern Andes. Geology, 1974, S. 603-604, 1974.

Heuberger, H.: Alpine Quaternary Glaciation. - Arctic and Alpine Environments, ed. J. D. Ives \& R. G. BARRY, S. 319-338, London 1974.

Fieusser, C. J.: Vegetation and Climate of the Southern Chilean Lake District During and Since the Last Interglaciation. - Quaternary Research, 4, S. 290-315, 1974.

HöllermanN, P.: Zur Frage der unteren Strukturbodengrenze in Gebirgen der Trockengebiete. - Z. Geomorph. N. F., Suppl. Bd. 15, S. 156-166, 1972 a.

- : Beiträge zur Problematik der rezenten Strukturbodengrenze. - Göttinger Geogr. Abh., 60 (H. Poser-Festschr.), S. 235-260, Göttingen 1972 b.

- : Probleme der rezenten geomorphologischen Höhenstufung im Rahmen einer vergleichenden Hochgebirgsgeographie. - Tagungsber. u. wiss. Abh. 40. dt. Geogr.Tag Innsbruck, S. 61-75, Wiesbaden 1976 a.

- : Ein außergewöhnliches „periglaziales“ Frostbodenvorkommen bei Bonn. - Decheniana, 129, S. 263-267, 1976 b.

- : Formen, Formengesellschaften und Untergrenzen in den heutigen periglazialen Höhenstufen der Hochgebirge Europas und Afrikas zwischen Subarktis und Aquator. Erdkde. 30, S. 300-302, 1976 c.

HövermanN, J.: Über glaziale und "periglaziale “ Erscheinungen in Erithrea und Nordabessinien. - In: Ergebnisse und Probleme moderner geographischer Forschung (Mortensen-Festschrift), Veröff. Akad. Raumfo'ci.. Landesplan., Abh. 28, S. 87-112, Bremen-Horn 1954.

- : Uber Verlauf und Gesetzmäßigkeit der Strukturbodengrenze. - Biul. Periglacjalny 11, S. 201-207, 1962.

Hope, G. S. \& Peterson, J. A.: Glaciation and Vegetation in the High New Guinea Mountains. - Quaternary Studies, ed. R. P. Suggate \& M. M. Cresswell, Roy. Soc. New Zealand, S. 155-162, Wellington 1975.

Houvenaghel, G. T.: Equatorial undercurrent and climate in the Galapagos Islands. - Nature, 2509, S. 565$566,1974$.

Huckriede, R.: Ein ${ }^{14} \mathrm{C}$-Wert für den Pluvial-Kalk von Sodiri, Kordofan, Republik Sudan. - Geologica et Palacontologica, 6, S. 173-175, 1972.

Jannsen, G.: Periglazialerscheinungen in Trockengebieten - ein vielschichtiges Problem. - Z. Geomorph. N. F., Suppl. Bd. 15, S. 167-176, 1972.
Kaiser, K., Kempf, E. K., Leroi-Gourhan, A. \& Schütt, H.: Quartärstratigraphische Untersuchungen aus dem Damaskus-Becken und seiner Umgebung. - Z. Geomorph. N. F., 17, S. 263-353, 1973.

Kersiaw, A. P.: Late Quaternary Vegetation and Climate in Northeastcrn Australia. - Quaternary Studies, ed. R. P. Suggate \& M. M. Cresswell, Roy. Soc. New Zealand, S. 181-187, Wellington 1975.

Khoвzi, J. \& Usselmann, P.: Problèmes de géomorphologie en Colombie. - Rev. Géogr. phys. Géol. dyn., XV, S. 193-206, 1973.

Klaus, D.: Die eiszeitlichen und nacheiszeitlichen Klimaschwankungen im zentralmexikanischen Hochland und ihre Ursachen. - Erdkde., 27, S. 180-192, 1973.

Klitsch, E., Sonntag, C., Weistroffer, K. \& El ShazLY, E. M.: Grundwasser der Zentralsahara: Fossile Vorräte. - Geol. Rdsch., 65, S. 264-287, 1976.

LAUER, W.: Klimatische Grundzüge der Höhenstufung tropischer Gebirge. - Tagungsber. u. wiss. Abh. 40. dt. Geogr.-Tay Innsbruck, S. 76-90, Wiesbaden 1976.

Livingstone, D. A.: Age of Deglaciation in the Ruwenzori Range, Uganda. - Nature, 194, S. 859-860, 1962.

LÖFfLER, E.: Beobachtungen zur periglazialen Höhenstufe in den Hochgebirgen von Papua New Guinea. - Erdkde., 29, S. 285-292, 1975.

MacNeish, R.: Early Man in the Andes. - Readings from Scientific American (New World Archaeology), S. 143153, San Francisco 1971.

Mercer, J. H.: The Allerød Oscillation: A European Climatic Anomaly? - Arctic and Alpine Research, 1, S. 227234, 1969.

- : Chilean glacial chronology 20,000-11,000 carbon-14 years ago: some global comparisons. - Science, 176, S. $1118-1120,1972$.

- : Cainozoic temperature trends in the southern hemisphere: Antarctic and Andean glacial evidence. - Palaeoecology of Africa, 8, S. 85-114, 1973.

- : Glacial History of Southernmost South America. Quaternary Research, 6, S. 125-166, 1976.

Messerli, B.: Formen und Formungsprozesse in der Hochgebirgsregion des Tibesti. - Hochgebirgsforschung, 2, S. 23-86, 1972.

- : Problems of vertical and horizontal arrangement in the high mountains of the extreme arid zone (central Sahara). - Arctic and Alpine Research, 5, S. A139-A147, 1973.

Michel, P.: Les bassins des fleuves Sénégal et Gambie. Etude géomorphologique. Tome 1, 2 u. 3 (Karten). Office de la Rech. Scient. Techn. Outre-Mer, Miém. ORSTOM No. 63, S. 1-752, Paris 1973.

Müller, P.: Biogéographie et évolution en Amérique du Sud. - C. R. Soc. Biogéogr. Séance 448, S. 15-22, 1974.

Patzelt, G.: Unterinntal - Zillertal - Pinzgau - Kitzbühel. Spät- und postglaziale Landschaftsentwidklung. Innsbrucker Geogr. Studien, 2, S. 309-329, 1975.

Richmond, G. M.: Appraisal of the Future Climate of the Holocene in the Rocky Mountains. - Quaternary Research, 2, S. 315-322, 1972.

Rohdenburg, H. \& SAbelberg, U.: Quartäre Klimazyklen im westlichen Mediterrangebiet und ihre Auswir- 
kungen auf die Relief- und Bodenentwicklung. - Catena, 1, S, 71-180, 1973.

Schubert, C.: Late Glacial Chronology in the Northeastern Venezuelan Andes. - 24th IGC, 1972, Sect. 12, S. 103-109, Montreal 1972.

- : Late Pleistocene Mérida Glaciation, Venezuelan Andes. - Boreas, 3, S. 147-152, 1974 a.

- : Late Pleistocene Glaciation of Páramo de La Culata, North-Central Venezuelan Andes. - Geol. Rdsch., 63, S. $516-538,1974$ b.

- : Striated ground on an arid tropical island: La Orchila, North-Central Venezuelan offshore. - Rev. Géomorph. dyn., XXIII, S. 27-31, 1974 c.

- : Glaciation and Periglacial Morphology in the Northwestern Venezuelan Andes. - Eiszeitalter u. Gegenwart, 26, S. 196-211, 1975.

Sears, P. B. \& Clisby, K. H.: Palynology in southern North America, Part IV: Pleistocene climate in Mexico. - Bull. Geol. Soc. Amer., 66, S. 521-530, 1955.

Sergin, S. Y. \& Chizhov, O. P.: Modeling of Global Changes of Climate and Glaciation in Pleistocene. International Geography '76: 2 (Climatology, Hydrology, Glaciology), XXIII Intern. Geogr. Cong. Moskau, S. $128-132$, Moskau 1976.

Shanzer, E. V., Petrov, O. M. \& Franco, G.: Sobre las formaciones costeras del Holoceno en Cuba, las terrazas Pleistocénicas de la región Habana-Matanzas y los sedimentos vinculados a ellas. - Serie Geología, 21, Acad. Cienc. Cuba, S. 1-26, La Habana 1975.

Singh, G. \& Agrawal, D. P.: Radiocarbon evidence for deglaciation in north-western Himalaya, India. - Nature, 260, S. 232, 1976.

Street, A. F. \& Grove, A. T.: Environmental and climatic implication of late Quaternary lake-level fluctuations in Africa. - Nature, 261, S. 385-389, 1976.

Suzuki, H.: Climatic zones of the Würm glacial stage. Bull. Dept. of Geogr., Univ. of Tokyo, 3, S. 35-46, 1971.

- : Recent and Würm Climates of the West Coast of South America. - Bull. Dept. of Geogr., Univ. of Tokyo, 5, S. 3-32, 1973.

Troll, C.: Die Stellung der Indianer-Hochkulturen im Landschaftsaufbau der tropischen Anden. - Ztschr. Ges. Erdkde. Berlin 1943, S. $93-128,1943$.
- : Strukturböden, Solifluktion und Frostklimate der Erde. - Geol. Rdsch., 34, S. 545-694, 1944.

Troll, C. \& PAfFen, K.H.: Die Jahreszeitenklimate der Erde. - Erdkde., 18, S. 5-28, 1964.

van der Hammen, T.: The Pleistocene changes of vegetation and climate in tropical South America. - J. Biogeogr., 1, S. 3-26, 1974

van Geel, B. \& van der Hammen, T.: Upper Quaternary vegetational and climatic sequence of the Fuquene area (Eastern Cordillera, Colombia). - Palaeogeogr. Palaeoclim. Palaeoecol., 14, S. 9-92, 1973.

van Zinderen Bakker, E. M.: Upper Pleistocene and Holocene stratigraphy and ecology on the basis of vegetation changes in sub-Saharan Africa. - Background to Evolution in Africa, ed. W. W. Bishop \& J. D. Clark, S. 125147, Chicago 1967.

- : The origin and palaeoenvironment of the Namib Desert biome. - J. Biogeogr., 2, S. 65-73, 1975.

van Zinderen Bakker, E. M. \& Coetzee, J. A.: A reappraisal of late-Quaternary climatic evidence from tropical Africa. - Palaeoecology of Africa, 7, S. 151-181, 1972.

WARREN, A.: Dune trends and their implications in the central Sudan. - Z. Geomorph. N. F., Suppl. Bd. 10, S. $154-180,1970$.

Washburn, A. L.: Periglacial processes and environments.S. 1-320, London 1973.

Weischet, W.: Zur Geomorphologie des Glatthang-Reliefs in der ariden Subtropenzone des Kleinen Nordens von Chile. Z. Geomorph. N. F., 13, S. 1-21, 1969.

WEYL, R.: Eiszeitliche Gletscherspuren in Costa Rica (Mittelamerika). - Ztschr. Gletscherkde. Glazialgeol., 3, S. 317 bis 325,1956

White, S. E.: Late Pleistocene Sequence for the West Side of Iztaccíhuatl, Mexico. - Bull. Geol. Soc. Amer., 73, S. 935-958, 1962.

Wilhelmy, H.: Eiszeit und Eiszeitklima in den feuchttropischen Anden. - Pet. Mitt. Erg. H. 262, S. 281-310, 1957.

Williams, M. A. J.: Radiocarbon dating and late Quaternary Saharan climates: a discussion. $-\mathrm{Z}$. Geomorph. N F., 20, S. 361-362, 1976.

WRIGHT, H. E.: Retreat of the Laurentide Ice Sheet From 14,000 to 9000 Years Ago. - Quaternary Research, 1, S. $316-330,1971$. 
Abb. 7 Die letzteiszeitlichen Maximal-Vergletscherungen in ihrer räumlichen und zeitlichen Verbreitung. Die Zonierung ist teilweise noch stark hypothetisch Maximal glaciations of the last Ice Age in their spatial and temporal distribution. In parts the zonation is still largely hypothetical.

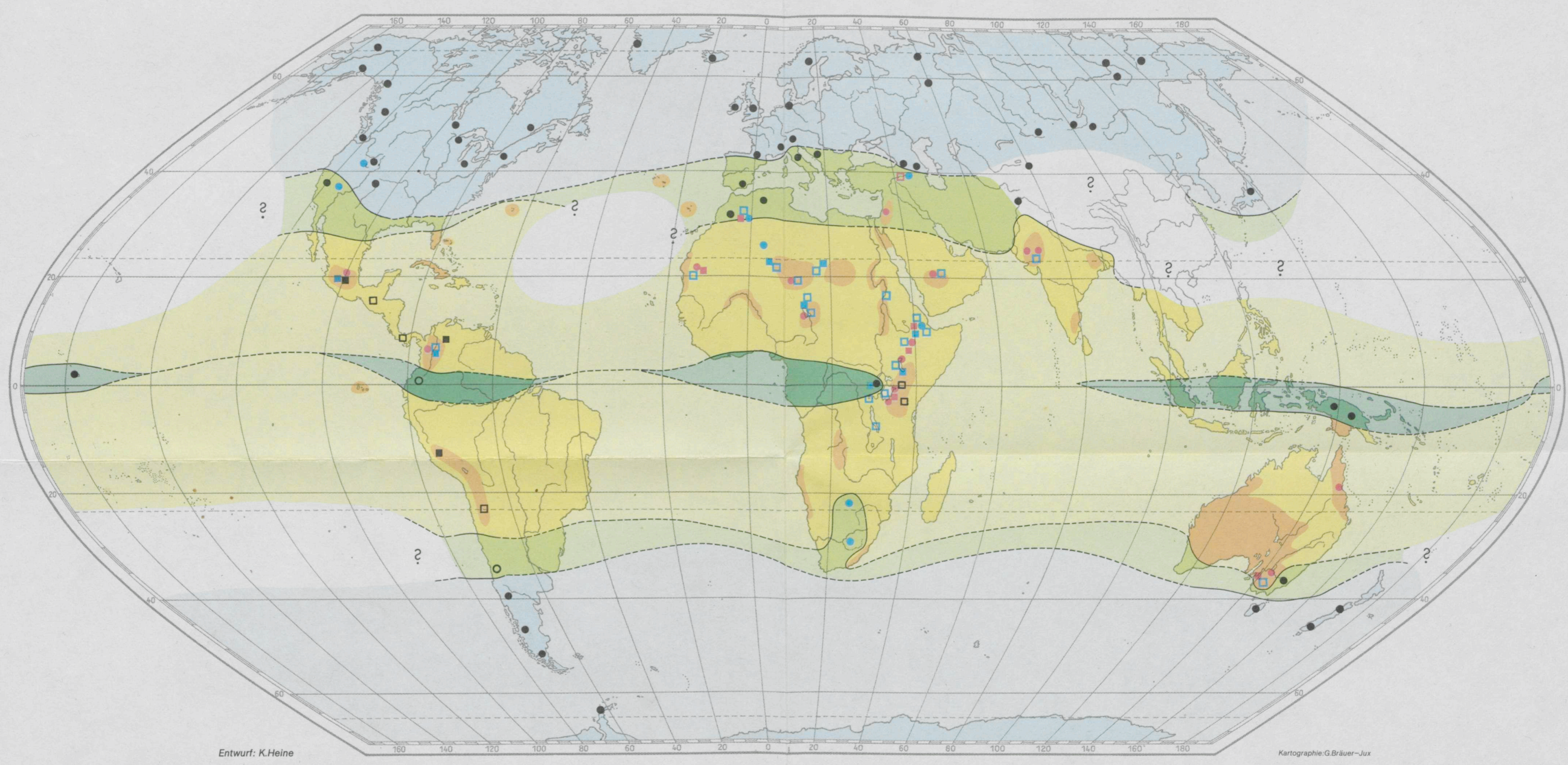

- datierte bzw. eindeutig stratigraphisch bestimmte hochglaziale Maximal -
Vergletscherung (ca. 20000-15000 BP)

- wahrscheinliche hochglaziale Maximal - Vergletscherung

$\square$ eindeutig belegte hochglaziale (extreme) Aridität

- datierte bzw. eindeutig stratigraphisch bestimmte spätglaziale Maximal -

口 wahrscheinliche bzw. vermutete spätglaziale Maximal-Vergletscherung

- hochglazialer Seespiegel-Hochstand
- spätglazialer Seespiegel - Hochstand (12000-11000 BP)

ㅁ. frühholozäner Seespiegel-Hochstand (9000-8000 BP)

- hochglazialer Seespiegel-Tiefstand

- spätglazialer Seespiegel - Tiefstand (12000-11000 BP)

ㅁ frühholozäner Seespiegel - Tiefstand (9000-8000 BP)

। $\square$ tropische Zone mit Maximal-Vergletscherung im Hochglazial infolge Temperaturabnahme bei-gegenuber heute - geringeren, jedoch für Gletscherbildunge
ausenden Niederschlägen " $\square \square$ glapisch- subtropische Zone mit aridem (bzw. gegenüber heute ariderem) hochglazial nur in der Sahara und den äthiopischen Gebirgen möglich (als Folge von Niederschlägen außertropischer, aus Norden eindringender Zyklonen). Für diese Zone ist eine spätglaziale Maximal-Vergletscherung charakteris
(niederschlagsabhăngige Schneegrenze) infolge großer spătglazialer hygrischer Schwankungen.

III $\square$ subtropisch - gemäßigte Zone mit gegenüber heute geringeren Niederschlägen im Hochglazial. Infolge starker Temperaturerniedrigung efrolgt die MaximalVergletscherung jedoch (wie in Zone I) im Hochglazial. Gebietsweise sind stär-
kere hygrische Schwankungen im Hoch--Spät- und Postglazial nachgewiesen.

V $\square$ außertropische Zone mit zum Teil - gegenüber heute - geringeren Niederschlägen,

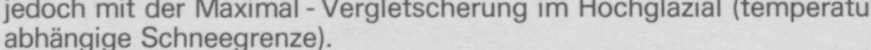

